Supporting Information for the Article:

\title{
Syntheses and Properties of Triruthenium Polyhydrido Complexes Composed of 1,2,4-tri-tert-butylcyclopentadienyl and $p$-Cymene Ruthenium Units
}

Toshiro Takao, * Hidenori Suzuki, and Ryuichi Shimogawa

Department of Chemical Science and Engineering, School of Materials and Chemical Technology, Tokyo Institute of Technology, 2-12-1 O-okayama, Meguro-ku, Tokyo 152-8552, Japan

Contents

1. Crystal data and results of XRD studies

S-2

2. Results of DFT calculations

S-7

3. NMR and IR spectra of compounds

S-15

4. Cyclic voltammograms of $\mathbf{3 a}, \mathbf{5}$, and $\mathbf{6}$

$\mathrm{S}-24$ 


\section{Crystal data and results of XRD studies}

Table S1. Crystallographic Data for 3a, 5, and 8 .

\begin{tabular}{|c|c|c|c|}
\hline & $3 \mathbf{a}$ & 5 & 8 \\
\hline \multicolumn{4}{|l|}{ (a) Crystal data } \\
\hline Empirical formula & $\mathrm{C}_{44} \mathrm{H}_{76} \mathrm{Ru}_{3}$ & $\mathrm{C}_{37} \mathrm{H}_{60} \mathrm{Ru}_{3}$ & $\mathrm{C}_{40} \mathrm{H}_{69} \mathrm{O}_{3} \mathrm{PRu}_{3}$ \\
\hline Formula weight & 908.25 & 808.06 & 932.13 \\
\hline Crystal description & Platelet & Needle & Platelet \\
\hline Crystal color & Blue & Brown & Brown \\
\hline Crystal size (mm) & $0.27 \times 0.24 \times 0.09$ & $0.15 \times 0.15 \times 0.08$ & $0.22 \times 0.11 \times 0.07$ \\
\hline Crystalizing solution & hexane $\left(-30{ }^{\circ} \mathrm{C}\right)$ & acetone $\left(-30^{\circ} \mathrm{C}\right)$ & methanol $\left(-30^{\circ} \mathrm{C}\right)$ \\
\hline Crystal system & Monoclinic & Monoclinic & Monoclinic \\
\hline Space group & $P 2_{1} / \mathrm{n}(\# 14)$ & $P 2_{1} / \mathrm{n}(\# 14)$ & $P 2_{1} / \mathrm{n}(\# 14)$ \\
\hline$a(\AA)$ & $10.4221(4)$ & $17.7767(10)$ & $10.0858(7)$ \\
\hline$b(\AA)$ & $20.3557(8)$ & $10.3209(6)$ & $26.8788(17)$ \\
\hline$c(\AA)$ & $20.3320(7)$ & $20.7709(14)$ & $15.2093(9)$ \\
\hline \multicolumn{4}{|l|}{$\alpha\left(^{\circ}\right)$} \\
\hline$\beta\left(^{\circ}\right)$ & $96.7920(10)$ & $103.895(2)$ & $95.798(7)$ \\
\hline \multicolumn{4}{|l|}{$\gamma\left({ }^{\circ}\right)$} \\
\hline Volume $\left(\AA^{3}\right)$ & 4283.1(3) & $3699.4(4)$ & $4102.1(5)$ \\
\hline$Z$ value & 4 & 4 & 4 \\
\hline$D_{\text {calc }}\left(\mathrm{g} / \mathrm{cm}^{3}\right)$ & 1.408 & 1.451 & 1.509 \\
\hline Measurement temp. $\left({ }^{\circ} \mathrm{C}\right)$ & -150 & -150 & -103 \\
\hline$\mu(\mathrm{MoK} \alpha)\left(\mathrm{mm}^{-1}\right)$ & 1.071 & 1.231 & 1.163 \\
\hline \multicolumn{4}{|l|}{ (b) Intensity measurements } \\
\hline Diffractometer & Rigaku R-AXIS & Rigaku R-AXIS & Rigaku R-AXIS \\
\hline Dirrractometer & RAPID & RAPID & RAPID \\
\hline Radiation & $\begin{array}{c}\operatorname{MoK} \alpha \\
(\lambda=0.71069 \AA)\end{array}$ & $\begin{array}{c}\operatorname{MoK} \alpha \\
(\lambda=0.71069 \AA)\end{array}$ & $\begin{array}{c}\operatorname{MoK} \alpha \\
(\lambda=0.71069 \AA)\end{array}$ \\
\hline Monochromator & Graphite & Graphite & Graphite \\
\hline $2 \theta \max \left({ }^{\circ}\right)$ & 50 & 55 & 55 \\
\hline Reflections collected & 56061 & 35543 & 62516 \\
\hline Independent reflections & $7834\left(R_{\mathrm{int}}=0.0809\right)$ & $8399\left(R_{\mathrm{int}}=0.1253\right)$ & $9395\left(R_{\mathrm{int}}=0.0538\right)$ \\
\hline Reflections observed $(>2 \sigma)$ & 6391 & 4724 & 8010 \\
\hline Abs. Correction type & Numerical & Empirical & Numerical \\
\hline Abs Transmission & $0.8056(\min )$ & 0.6807 (min) & 0.8137 (min) \\
\hline ADs. Iransmission & $0.9367(\max )$ & $1.0000(\max )$ & $0.8826(\max )$ \\
\hline (c) Refinement & SHELXL-2014/7 & SHELXL-2014/7 & SHELXL-2018/3 \\
\hline$R_{1}(I>2 \sigma(I))$ & 0.0399 & 0.0614 & 0.0373 \\
\hline$w R_{2}(I>2 \sigma(I))$ & 0.0949 & 0.1329 & 0.0883 \\
\hline$R_{1}$ (all data) & 0.0526 & 0.1343 & 0.0458 \\
\hline$w R_{2}$ (all data) & 0.1015 & 0.1962 & 0.0939 \\
\hline Data/Restraints/Parameters & $7834 / 0 / 461$ & 8399 / 0 / 386 & $9395 / 0 / 454$ \\
\hline GOF & 1.060 & 1.106 & 1.040 \\
\hline $\begin{array}{l}\text { Largest diff. Peak and hole } \\
\left(\mathrm{e} . \AA^{-3}\right)\end{array}$ & 0.893 and -1.609 & 1.501 and -2.010 & 1.389 and -0.914 \\
\hline CCDC deposition number & 2062053 & 2062054 & 2062055 \\
\hline
\end{tabular}




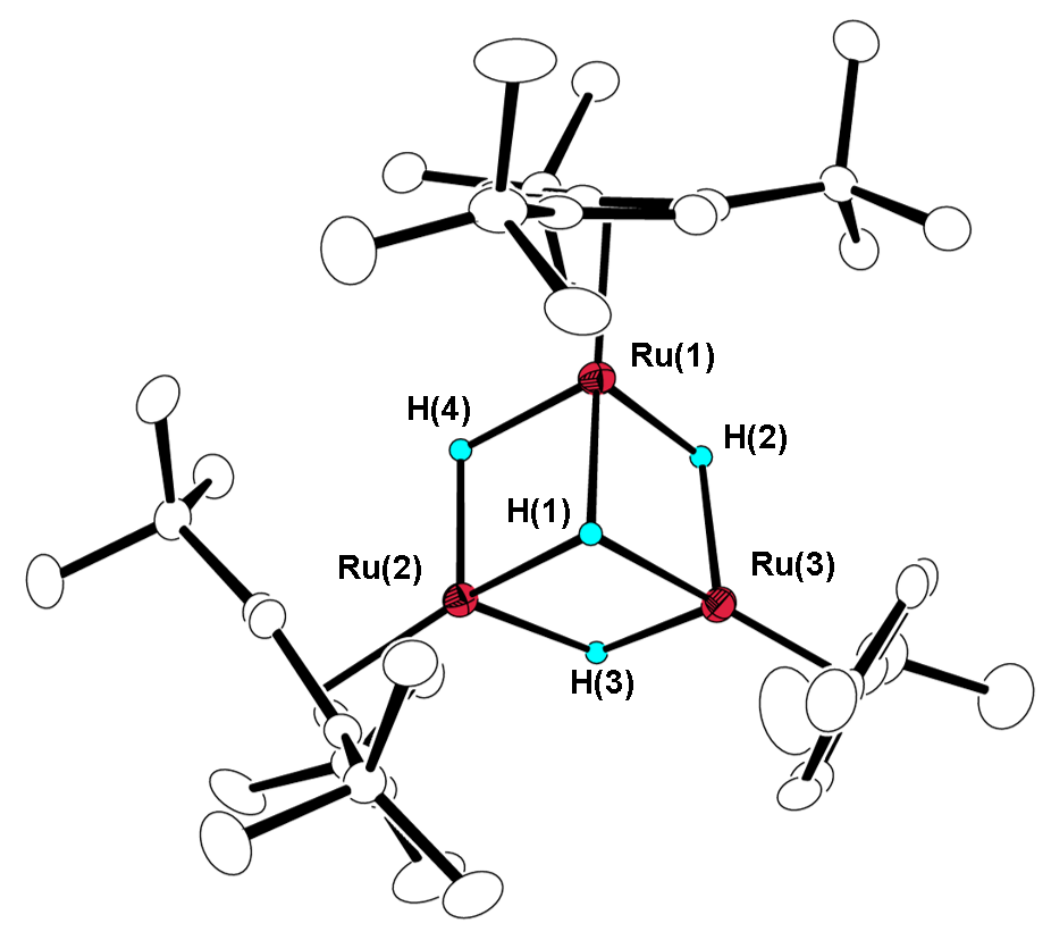

Figure S1. Molecular structure and labeling scheme of 3a with thermal ellipsoids at 30\% probability level. Hydrogen atoms except for those attached to Ru atoms were omitted for clarity.

Table S2. Selected bond lengths $[\AA]$ and angles $\left[^{\circ}\right]$ for $\mathbf{3 a}$.

Ru1 C3 2.153(4)

Ru1 C2 2.172(4)

Ru1 C1 2.190(4)

Ru1 C5 2.199(4)

Ru1 C4 2.220(4)

Ru1 Ru3 2.7104(5)

Ru1 Ru2 2.7430(5)

Ru1 H1 1.91(5)

Ru1 H2 1.65(5)

Ru1 H4 1.71(5)

Ru2 C20 2.163(4)

Ru2 C19 2.178(4)

Ru2 C22 2.185(4)

Ru2 C18 2.195(4)

Ru2 C21 2.199(4)

Ru2 Ru3 2.7260(5)

Ru2 H1 1.88(5)

Ru2 H3 1.78(6)

Ru2 H4 1.64(5)

Ru3 C38 2.194(5)

Ru3 C37 2.200(5)

Ru3 C35 2.202(4)

Ru3 C36 2.216(4)

Ru3 C39 2.226(5)
Ru3 C40 2.239(4)

Ru3 H1 1.90(5)

Ru3 H2 1.75(5)

Ru3 H3 1.63(6)

Ru3 Ru1 Ru2 59.978(12)

Ru3 Ru1 H1 44.6(14)

Ru2 Ru1 H1 43.3(15)

Ru3 Ru1 H2 38.5(18)

Ru2 Ru1 H2 85.4(18)

H1 Ru1 H2 83(2)

Ru3 Ru1 H4 90.4(18)

Ru2 Ru1 H4 34.2(18)

H1 Ru1 H4 76(2)

H2 Ru1 H4 102(3)

Ru3 Ru2 Ru1 59.419(12)

Ru3 Ru2 H1 44.3(15)

Ru1 Ru2 H1 44.0(15)

Ru3 Ru2 H3 35.0(18)

Ru1 Ru2 H3 82.8(18)

H1 Ru2 H3 79(2)

Ru3 Ru2 H4 91.3(18)

Ru1 Ru2 H4 35.9(19)

H1 Ru2 H4 78(2)
H3 Ru2 H4 102(3)

Ru1 Ru3 Ru2 60.603(12)

Ru1 Ru3 H1 44.7(14)

Ru2 Ru3 H1 43.7(15)

Ru1 Ru3 H2 35.8(17)

Ru2 Ru3 H2 84.0(17)

H1 Ru3 H2 80(2)

Ru1 Ru3 H3 86(2)

Ru2 Ru3 H3 39(2)

H1 Ru3 H3 83(2)

H2 Ru3 H3 90(3) 
(2) $\left[\left(\mathrm{Cp}{ }^{\dagger} \mathrm{Ru}\right)\{(p \text {-cymene }) \mathrm{Ru}\}_{2}(\mu-\mathrm{H})_{2}\left(\mu_{3}-\mathrm{H}\right)\right](\mathbf{5})$

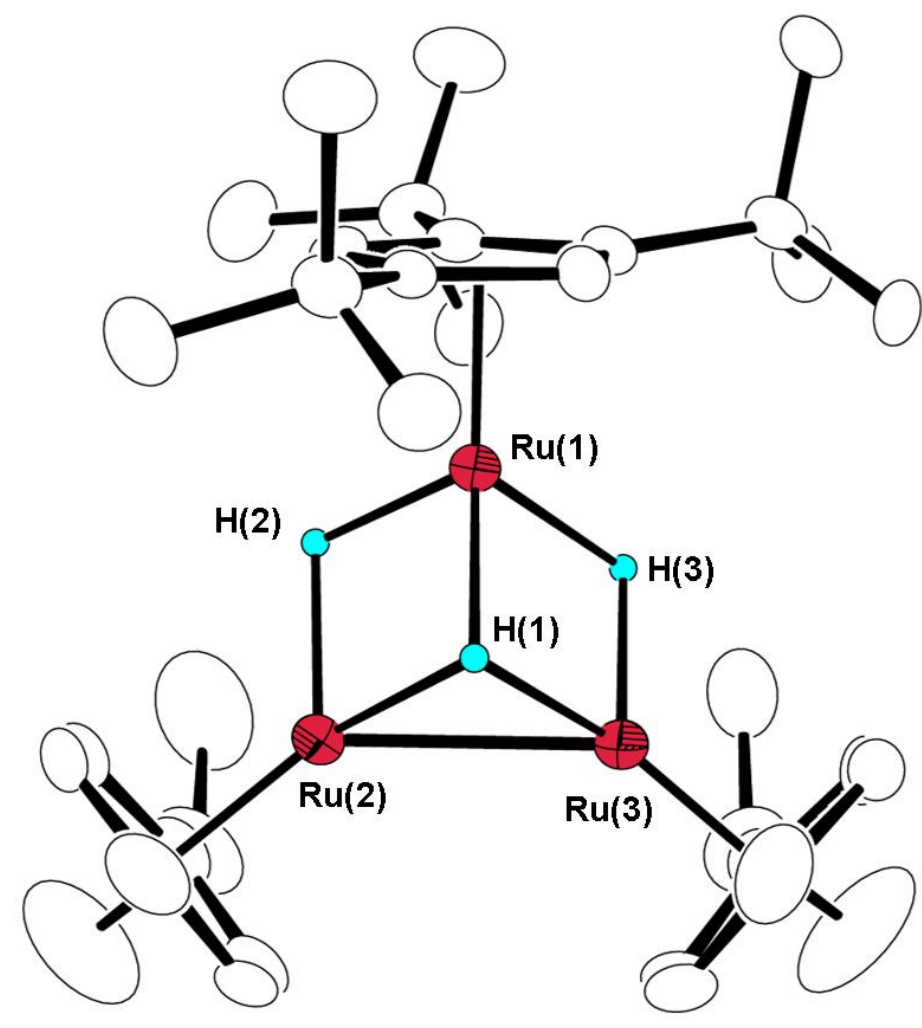

Figure S2. Molecular structure and labeling scheme of a tetrafluoroborate salt of $\mathbf{5}$ with thermal ellipsoids at $30 \%$ probability level. Hydrogen atoms except for those attached to $\mathrm{Ru}$ atoms were omitted for clarity.

Table S3. Selected bond lengths $[\AA]$ and angles $\left[^{\circ}\right]$ for $\mathbf{5}$.

Ru1 C2 2.169(8)

Ru1 C3 2.171(8)

Ru1 C5 2.179(8)

Ru1 C4 2.193(8)

Ru1 C1 2.199(7)

Ru1 Ru3 2.6747(9)

Ru1 Ru2 2.6873(9)

Ru1 H1 1.83(14)

Ru1 H2 1.64(14)

Ru1 H3 1.82(14)

Ru2 C20 2.184(9)

Ru2 C18 2.189(9)

Ru2 C22 2.210(10)
Ru2 C21 2.215(9)

Ru2 C19 2.222(9)

Ru2 C23 2.223(8)

Ru2 Ru3 2.5942(10)

Ru2 H1 1.86(13)

Ru2 H2 1.74(14)

Ru3 C31 2.156(9)

Ru3 C32 2.179(9)

Ru3 C28 2.190(10)

Ru3 C33 2.207(10)

Ru3 C30 2.230(8)

Ru3 C29 2.247(9)

Ru3 H1 1.79(14)
Ru3 H3 1.72(14)

Ru3 Ru1 Ru2 57.87(2)

Ru3 Ru1 H1 42(4)

Ru2 Ru1 H1 44(4)

Ru3 Ru1 H2 92(5)

Ru2 Ru1 H2 39(5)

H1 Ru1 H2 81(6)

Ru3 Ru1 H3 40(4)

Ru2 Ru1 H3 85(4)

H1 Ru1 H3 81(6)

H2 Ru1 H3 103(6)

Ru3 Ru2 Ru1 60.82(2)
Ru3 Ru2 H1 44(4)

Ru1 Ru2 H1 43(4)

Ru3 Ru2 H2 93(5)

Ru1 Ru2 H2 36(5)

H1 Ru2 H2 78(6)

Ru2 Ru3 Ru1 61.31(2)

Ru2 Ru3 H1 46(4)

Ru1 Ru3 H1 43(5)

Ru2 Ru3 H3 90(5)

Ru1 Ru3 H3 43(5)

H1 Ru3 H3 85(7) 
(3) $\left[(\mathrm{Cp}: \mathrm{Ru})\{(p-c y m e n e) \mathrm{Ru}\}_{2}(\mu-\mathrm{H})_{3}\left\{\mathrm{P}(\mathrm{OMe})_{3}\right\}\right](8)$

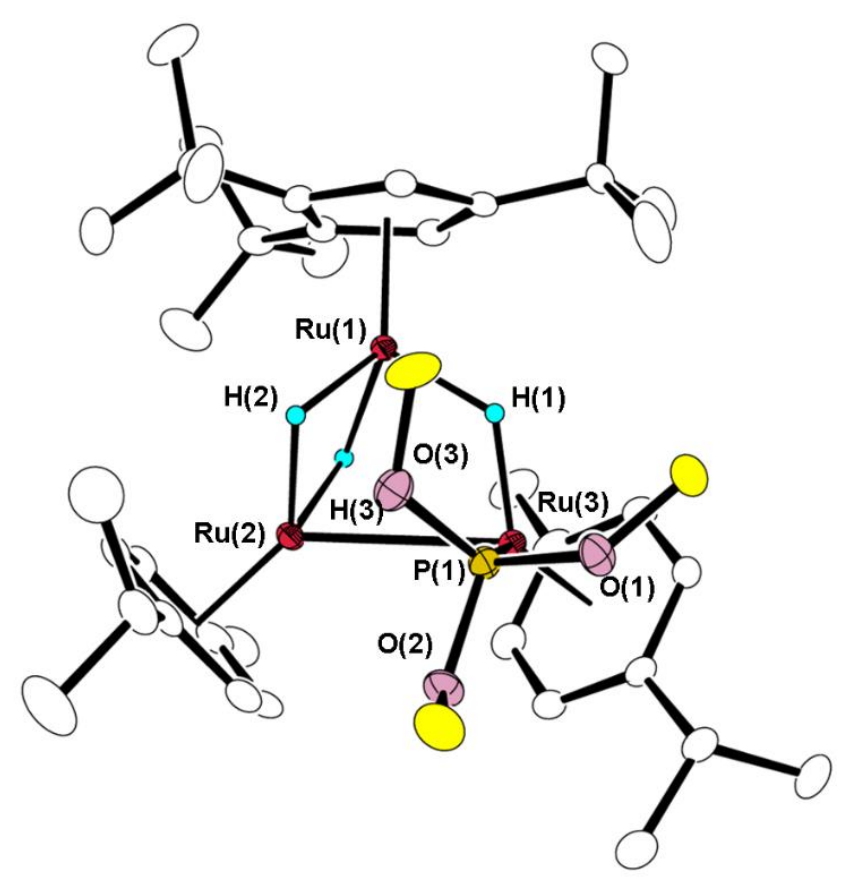

Figure S3. Molecular structure and labeling scheme of a tetrafluoroborate salt of $\mathbf{8}$ with thermal ellipsoids at $30 \%$ probability level. Hydrogen atoms except for those attached to Ru atoms were omitted for clarity.

Table S4. Selected bond lengths $[\AA]$ and angles $\left[^{\circ}\right]$ for $\mathbf{8}$.

$\begin{array}{lll}\text { Ru1 Ru2 2.6570(4) } & \text { O1 C1 1.427(4) } & \text { Ru2 Ru3 Ru1 54.700(9) } \\ \text { Ru1 Ru3 2.9804(4) } & \text { O2 C2 1.432(4) } & \text { Ru1 Ru2 Ru3 66.271(10) } \\ \text { Ru3 P1 2.1971(9) } & \text { O3 C3 1.415(5) } & \text { O2 P1 O3 97.17(14) } \\ \text { Ru3 Ru2 2.7914(4) } & & \text { O2 P1 O1 96.35(13) } \\ \text { P1 O2 1.615(3) } & \text { Ru2 Ru1 Ru3 59.029(9) } & \text { O3 P1 O1 98.99(14) } \\ \text { P1 O3 1.617(3) } & \text { P1 Ru3 Ru2 85.47(2) } & \text { O2 P1 Ru3 115.71(10) } \\ \text { P1 O1 1.637(2) } & \text { P1 Ru3 Ru1 98.22(3) } & \text { O3 P1 Ru3 125.59(10) }\end{array}$

O1 P1 Ru3 117.51(10)

C1 O1 P1 124.0(2)

C2 O2 P1 122.1(3)

C3 O3 P1 120.9(3) 
(4) Preliminary result of XRD study for $\left[\left(\mathrm{Cp}{ }^{\ddagger} \mathrm{Ru}\right)_{2}\{(p\right.$-cymene $\left.) \mathrm{Ru}\}(\mu-\mathrm{H})_{4}\left\{\mathrm{P}(\mathrm{OMe})_{3}\right\}\right](7)$

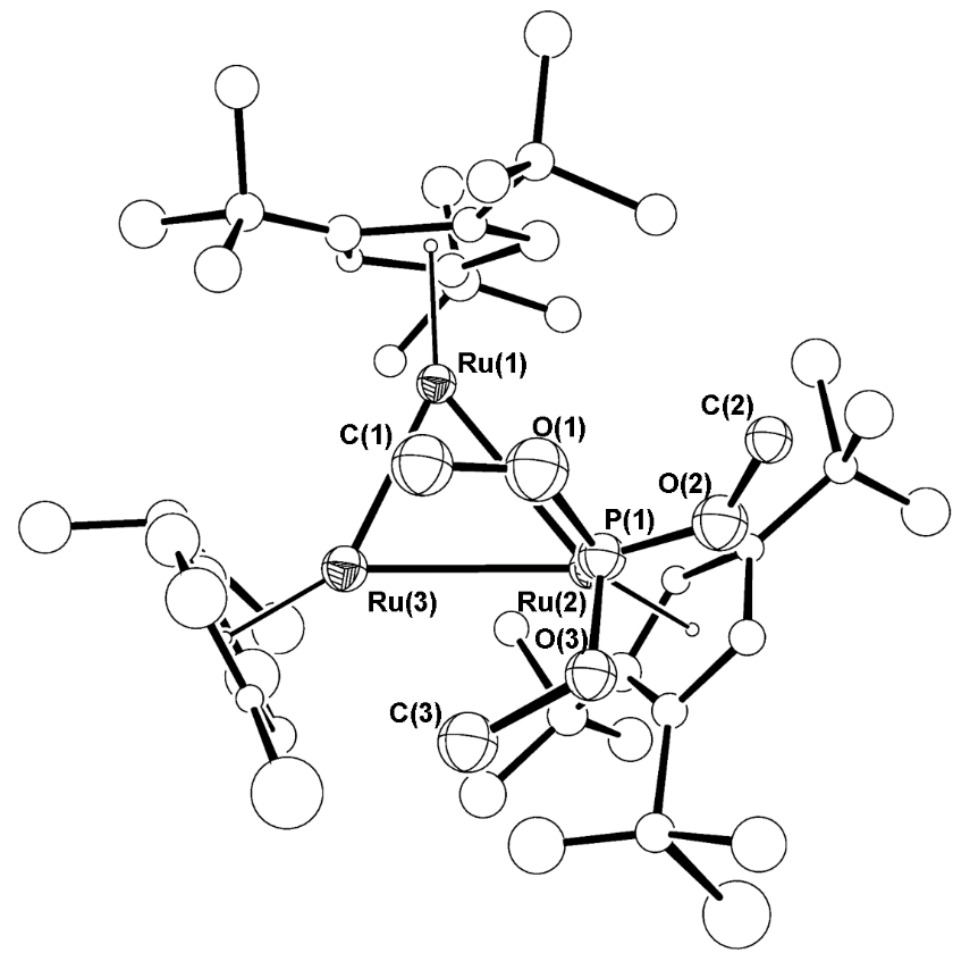

Figure S4. Molecular structure and labeling scheme of $\left[(\mathrm{Cp} \mathrm{Ru})_{2}\{(p\right.$-cymene $\left.) \mathrm{Ru}\}(\mu-\mathrm{H})_{4}\left\{\mathrm{P}(\mathrm{OMe})_{3}\right\}\right]$ (7) with thermal ellipsoids at 30\% probability level (preliminary result). All atoms were refined isotropically and hydrogen atoms were not located due to the low quality of the crystal. Selected crystal data: triclinic, $P 1, a=11.6267(8), b=11.6289(8), c=18.2136(13), \alpha=90.772(6), \beta=90.765(6), \gamma=$ 93.412(7), $Z=2, R_{1}=0.271, w R_{2}=0.5964, \mathrm{GOF}=3.038$. Selected bond lengths $(\AA): \operatorname{Ru}(1)-\operatorname{Ru}(2)$ 3.042(5), $\mathrm{Ru}(1)-\mathrm{Ru}(3)$ 2.620(5), $\mathrm{Ru}(2)-\mathrm{Ru}(3)$ 3.065(5), $\mathrm{Ru}(2)-\mathrm{P}(1) 2.248(14)$. 


\section{Results of DFT calculations}

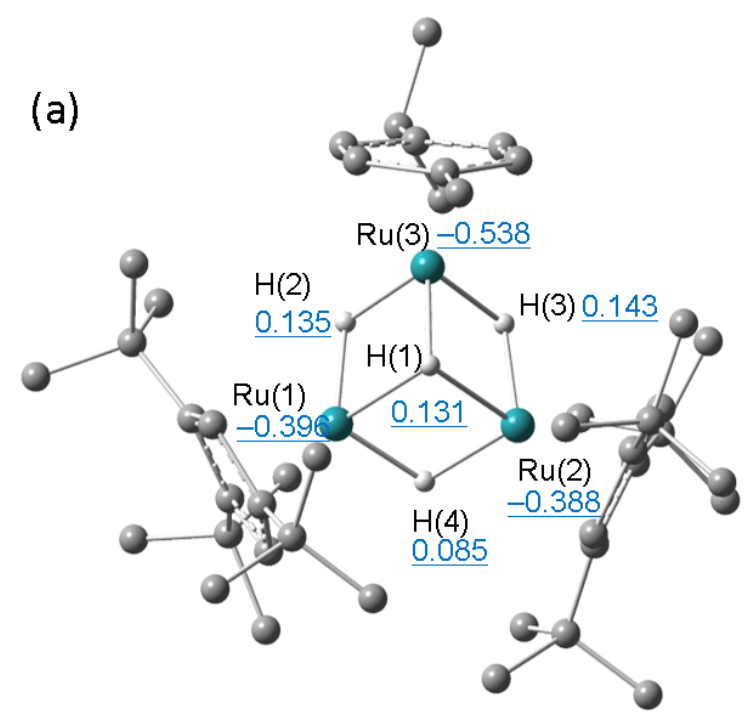

(b)

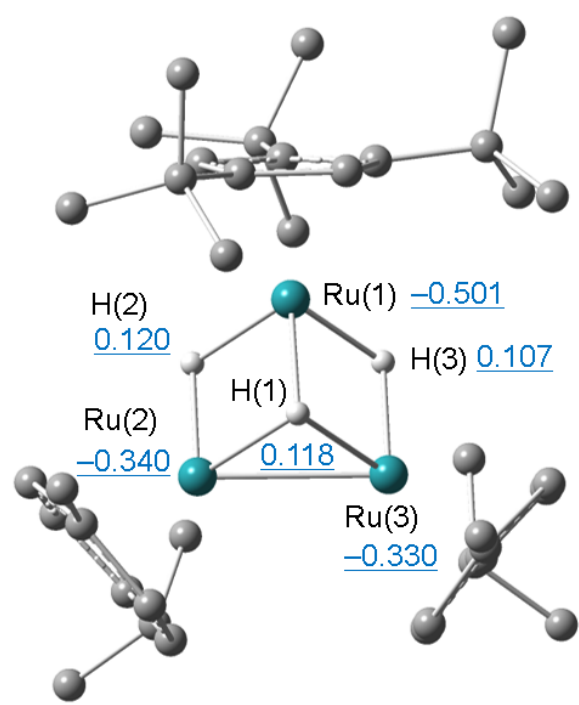

Figure S5. Optimized structures of (a) 3a and (b) $\mathbf{5}$ with NBO charges at the selected atoms $(\omega \mathrm{B} 97 \mathrm{XD} / \mathrm{LanL} 2 \mathrm{DZ}(\mathrm{Ru}), 6-31++\mathrm{G}(\mathrm{C}$ and $\mathrm{H}))$. Hydrogen atoms attached to $\mathrm{Cp}^{\ddagger}$ and $p$-cymene groups were omitted for clarity.

Table S5. Selected geometrical parameters for the optimized structures $\mathbf{3 a}$ and $\mathbf{5}$ with observed values.

\begin{tabular}{c||c|c||c||c|c}
\hline \multicolumn{3}{c||}{ 3a } & \multicolumn{3}{|c}{$\mathbf{5}$} \\
\hline & (opt.) & (obsd.) & & (opt.) & (obsd.) \\
\hline $\mathrm{Ru}(1)-\mathrm{Ru}(2)$ & 2.7508 & $2.7430(5)$ & $\mathrm{Ru}(1)-\mathrm{Ru}(2)$ & 2.6872 & $2.6873(9)$ \\
$\mathrm{Ru}(1)-\mathrm{Ru}(3)$ & 2.7201 & $2.7104(5)$ & $\mathrm{Ru}(1)-\mathrm{Ru}(3)$ & 2.6900 & $2.6747(9)$ \\
$\mathrm{Ru}(2)-\mathrm{Ru}(3)$ & 2.7268 & $2.7260(5)$ & $\mathrm{Ru}(2)-\mathrm{Ru}(3)$ & 2.5584 & $2.5942(10)$ \\
$\mathrm{Ru}(1)-\mathrm{H}(1)$ & 1.939 & $1.91(5)$ & $\mathrm{Ru}(1)-\mathrm{H}(1)$ & 1.951 & $1.83(14)$ \\
$\mathrm{Ru}(1)-\mathrm{H}(2)$ & 1.767 & $1.65(5)$ & $\mathrm{Ru}(1)-\mathrm{H}(2)$ & 1.739 & $1.64(14)$ \\
$\mathrm{Ru}(1)-\mathrm{H}(4)$ & 1.739 & $1.71(5)$ & $\mathrm{Ru}(1)-\mathrm{H}(4)$ & 1.740 & $1.82(14)$ \\
$\mathrm{Ru}(2)-\mathrm{H}(1)$ & 1.961 & $1.88(5)$ & $\mathrm{Ru}(2)-\mathrm{H}(1)$ & 1.889 & $1.86(13)$ \\
$\mathrm{Ru}(2)-\mathrm{H}(3)$ & 1.757 & $1.78(6)$ & $\mathrm{Ru}(2)-\mathrm{H}(2)$ & 1.762 & $1.74(14)$ \\
$\mathrm{Ru}(2)-\mathrm{H}(4)$ & 1.736 & $1.64(5)$ & & & \\
$\mathrm{Ru}(3)-\mathrm{H}(1)$ & 1.805 & $1.90(5)$ & $\mathrm{Ru}(3)-\mathrm{H}(1)$ & 1.883 & $1.79(14)$ \\
$\mathrm{Ru}(3)-\mathrm{H}(2)$ & 1.714 & $1.75(5)$ & & & 1.752 \\
$\mathrm{Ru}(3)-\mathrm{H}(3)$ & 1.713 & $1.63(6)$ & $\mathrm{Ru}(3)-\mathrm{H}(3)$ & & $1.72(14)$ \\
$\mathrm{Ru}(2)-\mathrm{Ru}(1)-\mathrm{Ru}(3)$ & 59.787 & $59.978(12)$ & $\mathrm{Ru}(2)-\mathrm{Ru}(1)-\mathrm{Ru}(3)$ & 56.821 & $57.87(2)$ \\
$\mathrm{Ru}(1)-\mathrm{Ru}(2)-\mathrm{Ru}(3)$ & 59.548 & $59.419(12)$ & $\mathrm{Ru}(1)-\mathrm{Ru}(2)-\mathrm{Ru}(3)$ & 61.644 & $60.82(2)$ \\
$\mathrm{Ru}(1)-\mathrm{Ru}(3)-\mathrm{Ru}(2)$ & 60.665 & $60.603(12)$ & $\mathrm{Ru}(1)-\mathrm{Ru}(3)-\mathrm{Ru}(2)$ & 61.535 & $61.31(2)$ \\
\hline \hline
\end{tabular}


(a)

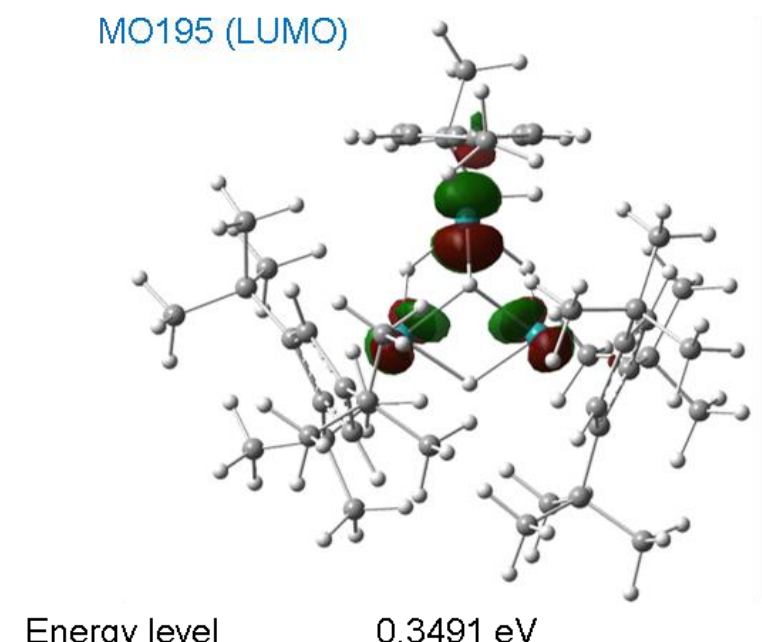

Energy level

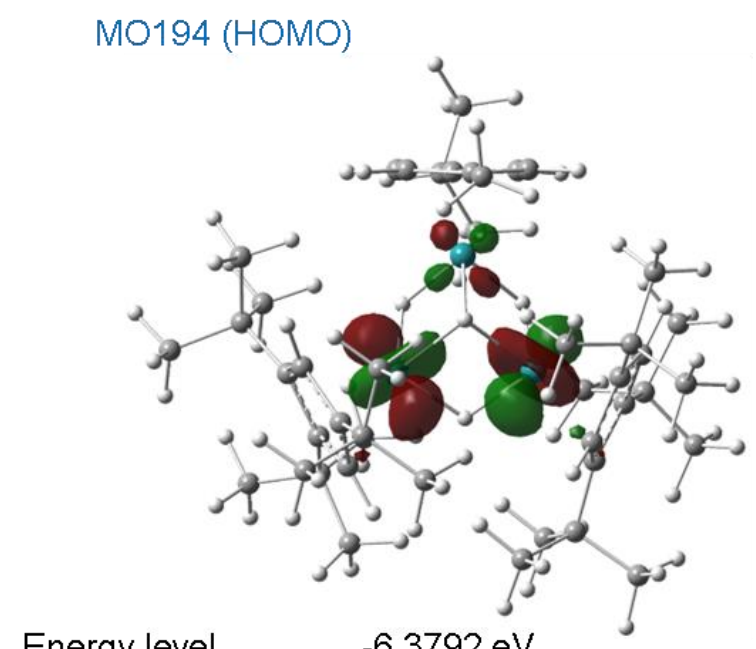

(b)
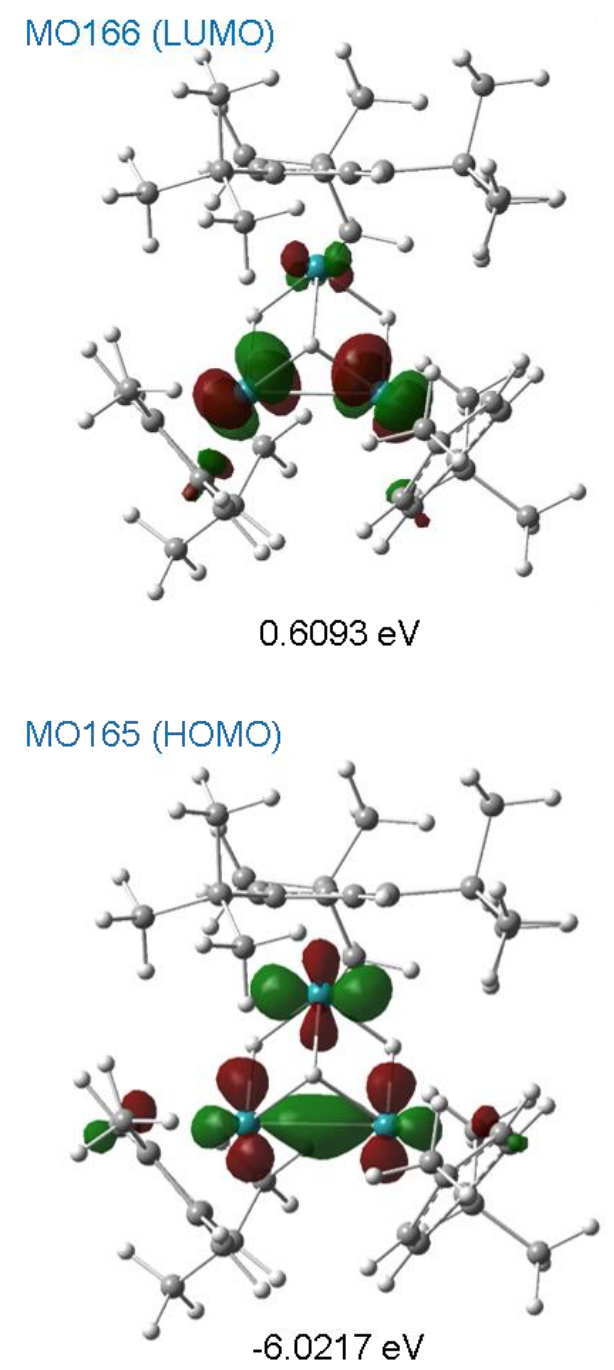

Figure S6. The shapes of LUMO and HOMO of (a) 3a and (b) 5 showing with their Eigenvalues (eV) $(\omega \mathrm{B} 97 \mathrm{XD} / \mathrm{LanL} 2 \mathrm{DZ}(\mathrm{Ru}), 6-31++\mathrm{G}(\mathrm{C}$ and $\mathrm{H})$, isovalue 0.06$)$. 


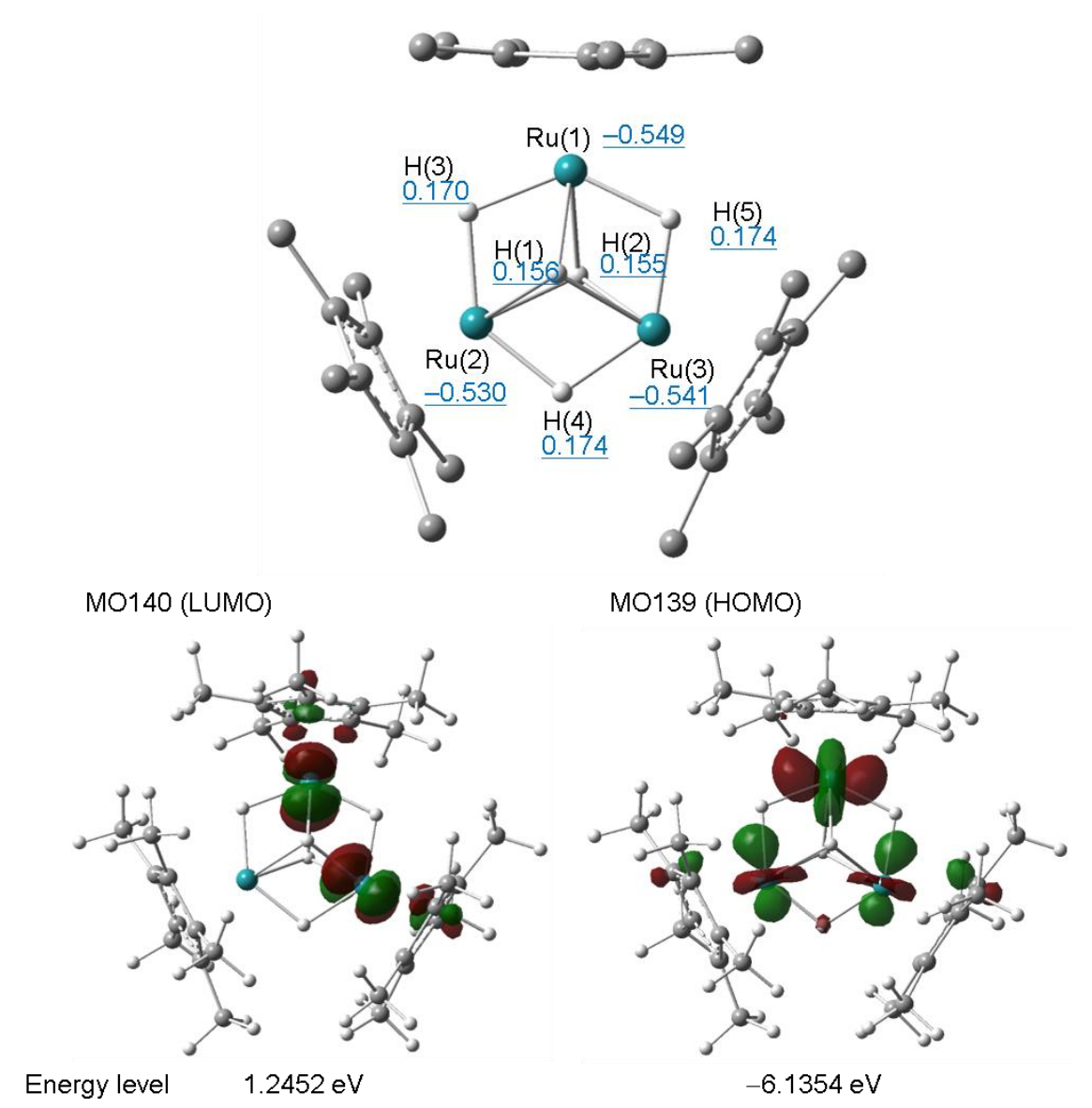

Figure S7. Optimized structures of $\left[\{\mathrm{Cp} * \mathrm{Ru}(\mu-\mathrm{H})\}_{3}\left(\mu_{3}-\mathrm{H}\right)_{2}\right](6)$ with $\mathrm{NBO}$ charges at the selected atoms and the shapes of LUMO and HOMO with their Eigenvalues $(\mathrm{eV})(\omega \mathrm{B} 97 \mathrm{XD} / \mathrm{LanL} 2 \mathrm{DZ}(\mathrm{Ru})$, 6-311++G(d,p) $(\mathrm{RuH}), 6-31 \mathrm{G}(\mathrm{d})(\mathrm{C}$ and $\mathrm{CH})$, isovalue 0.06$)$.

Table S6. Selected geometrical parameters for the optimized structure of $\mathbf{6}$ and observed data.

\begin{tabular}{l||c||c}
\hline & 6 (opt.) & 6 (obsd.) \\
\hline $\mathrm{Ru}(1)-\mathrm{Ru}(2)$ & 2.7896 & $2.7534(8)$ \\
$\mathrm{Ru}(1)-\mathrm{Ru}(3)$ & 2.7867 & $2.7503(8)$ \\
$\mathrm{Ru}(2)-\mathrm{Ru}(3)$ & 2.7907 & $2.7453(6)$ \\
$\mathrm{Ru}(1)-\mathrm{H}(1)$ & 1.959 & $1.94(3)$ \\
$\mathrm{Ru}(1)-\mathrm{H}(2)$ & 1.981 & $1.94(3)$ \\
$\mathrm{Ru}(1)-\mathrm{H}(3)$ & 1.726 & $1.67(2)$ \\
$\mathrm{Ru}(1)-\mathrm{H}(5)$ & 1.732 & $1.66(2)$ \\
$\mathrm{Ru}(2)-\mathrm{H}(1)$ & 2.011 & $1.94(3)$ \\
$\mathrm{Ru}(2)-\mathrm{H}(2)$ & 1.948 & $1.94(3)$ \\
$\mathrm{Ru}(2)-\mathrm{H}(3)$ & 1.729 & $1.65(2)$ \\
$\mathrm{Ru}(2)-\mathrm{H}(4)$ & 1.731 & $1.66(2)$ \\
$\mathrm{Ru}(3)-\mathrm{H}(1)$ & 1.947 & $1.94(3)$ \\
$\mathrm{Ru}(3)-\mathrm{H}(2)$ & 1.982 & $1.94(3)$ \\
$\mathrm{Ru}(3)-\mathrm{H}(4)$ & 1.733 & $1.65(2)$ \\
$\mathrm{Ru}(3)-\mathrm{H}(5)$ & 1.730 & $1.65(2)$ \\
& & \\
$\mathrm{Ru}(2)-\mathrm{Ru}(1)-\mathrm{Ru}(3)$ & 60.059 & $59.844(18)$ \\
$\mathrm{Ru}(1)-\mathrm{Ru}(2)-\mathrm{Ru}(3)$ & 59.919 & $60.02(2)$ \\
$\mathrm{Ru}(1)-\mathrm{Ru}(3)-\mathrm{Ru}(2)$ & 60.022 & $60.135(18)$ \\
\hline
\end{tabular}

${ }^{a}$ Suzuki, H.; Kakigano, T.; Tada, K.; Igarashi, M.; Matsubara, K.; Inagaki, A.; Oshima, M.; Takao, T., Bull. Chem. Soc. Jpn. 2005, 78, 67-87. 
(a)
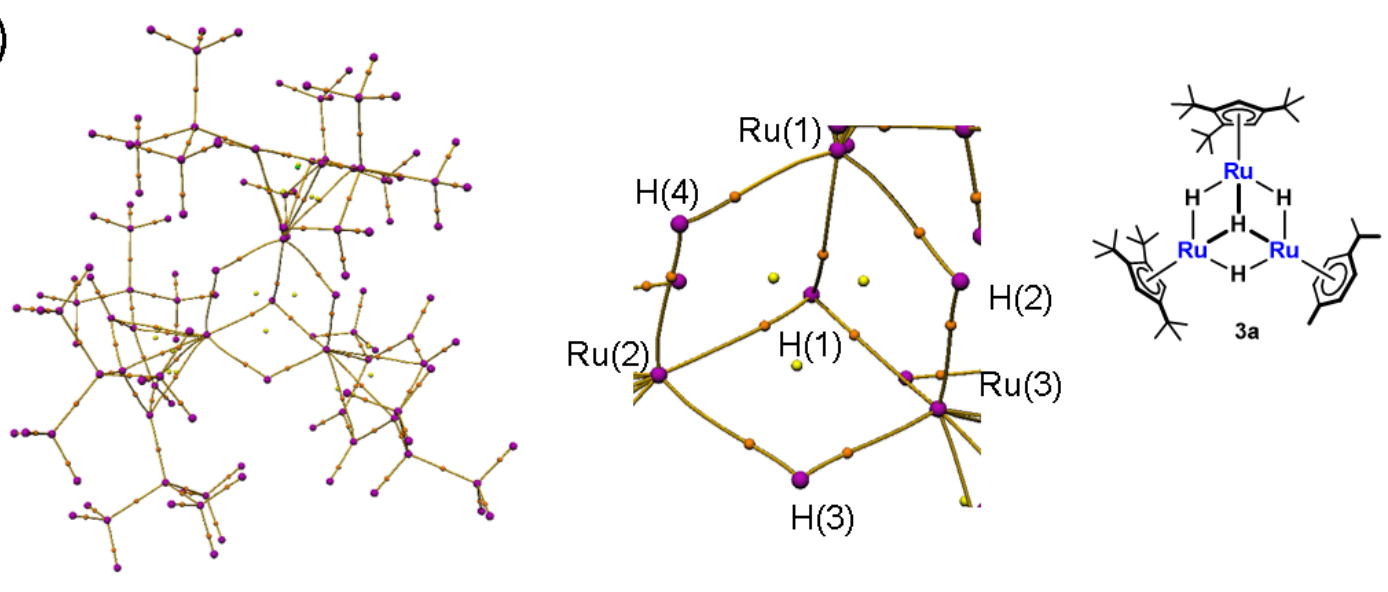

(b)
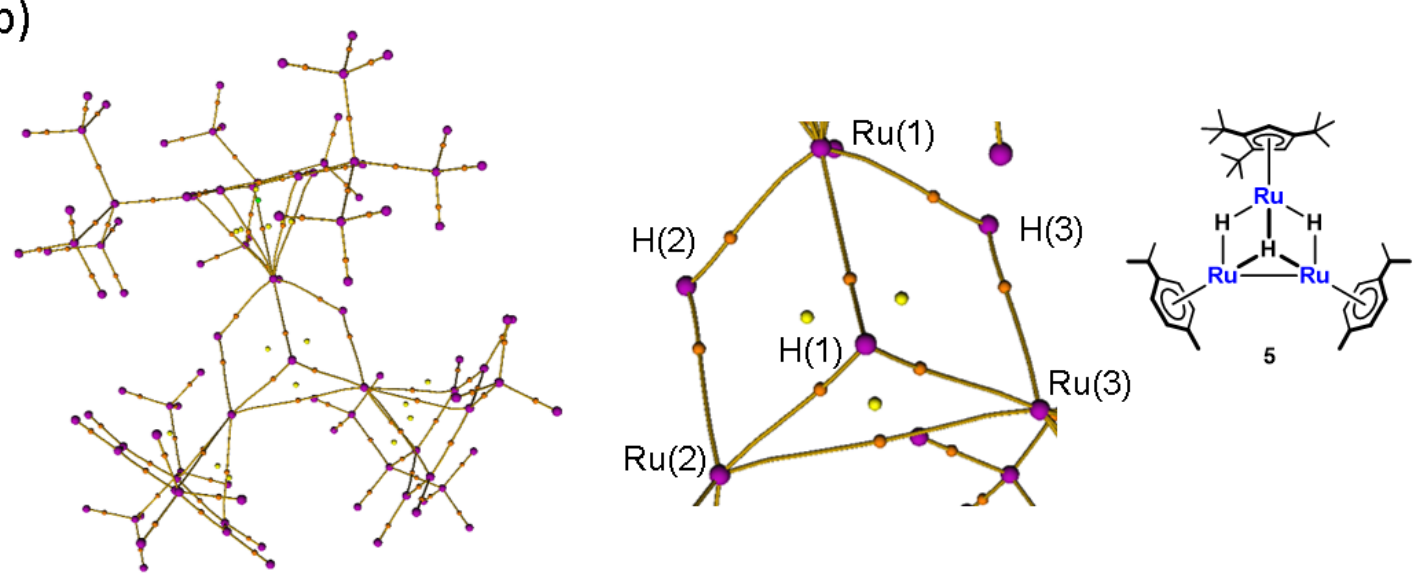

(c)
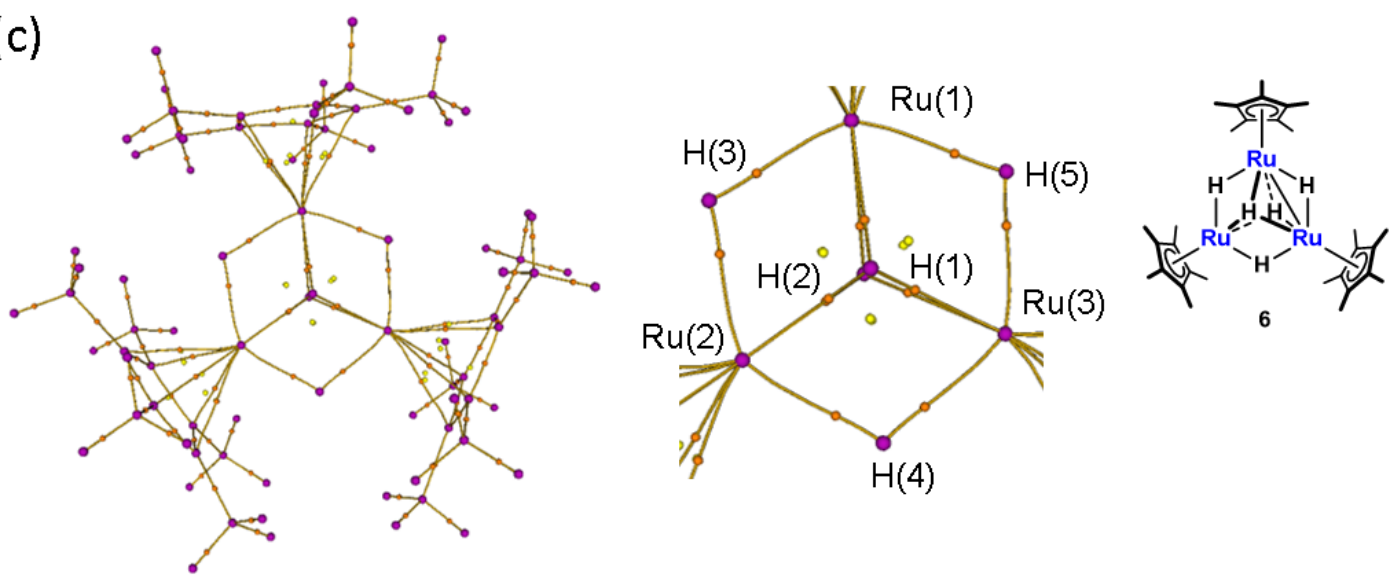

Figure S8. Optimized structures and enlarged view of the $\mathrm{Ru}_{3}$ core of (a) $\mathbf{3 a}$, (b) 5, and (c) $\mathbf{6}$, showing bond paths (orange lines), the bond critical points (small orange circles), and the ring critical points small yellow circles). 

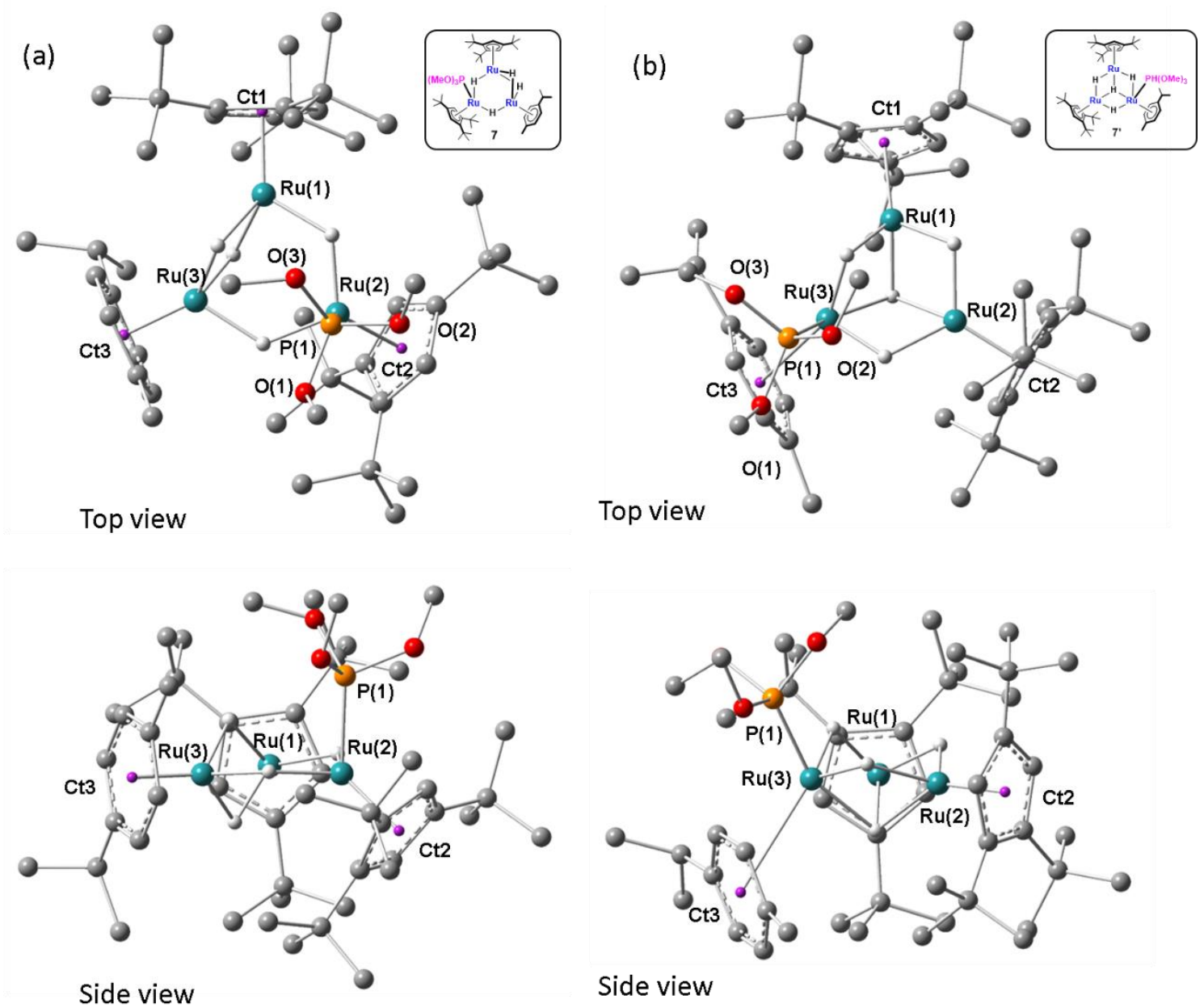

Figure S9. Optimized structures of (a) $\left[(\mathrm{Cp} * \mathrm{Ru})_{2}\{(p\right.$-cymene $\left.) \mathrm{Ru}\}(\mu-\mathrm{H})_{4} \mathrm{P}(\mathrm{OMe})_{3}\right](7)$ and (b) possible isomer 7' $(\omega \mathrm{B} 97 \mathrm{XD} / \mathrm{SDD}(\mathrm{Ru}), 6-31++\mathrm{G}(\mathrm{C}, \mathrm{H}, \mathrm{O}$, and $\mathrm{P}))$. Hydrogen atoms except for attached to $\mathrm{Ru}$ atoms were omitted for clarity.

Table S7. Selected geometrical parameters for the optimized structures of $\mathbf{7}$ and $\mathbf{7}$ ' and relative energies between them.

\begin{tabular}{c||c||c||c}
\hline & $\mathbf{7}$ (opt.) & & $\mathbf{7}^{\prime}$ (opt.) \\
\hline $\mathrm{Ru}(1)-\mathrm{Ru}(2)$ & 3.0250 & $\mathrm{Ru}(1)-\mathrm{Ru}(2)$ & 2.6781 \\
$\mathrm{Ru}(1)-\mathrm{Ru}(3)$ & 2.6995 & $\mathrm{Ru}(1)-\mathrm{Ru}(3)$ & 2.6640 \\
$\mathrm{Ru}(2)-\mathrm{Ru}(3)$ & 3.0765 & $\mathrm{Ru}(2)-\mathrm{Ru}(3)$ & 2.8145 \\
$\mathrm{Ru}(2)-\mathrm{P}(1)$ & 2.2038 & $\mathrm{Ru}(2)-\mathrm{P}(1)$ & 2.1850 \\
$\mathrm{P}(1)-\mathrm{O}(1)$ & 1.6525 & $\mathrm{P}(1)-\mathrm{O}(1)$ & 1.6282 \\
$\mathrm{P}(1)-\mathrm{O}(2)$ & 1.6421 & $\mathrm{P}(1)-\mathrm{O}(2)$ & 1.6228 \\
$\mathrm{P}(1)-\mathrm{O}(3)$ & 1.6480 & $\mathrm{P}(1)-\mathrm{O}(3)$ & 1.6530 \\
$\mathrm{Ru}(1)-\mathrm{Ct} 1$ & 1.797 & $\mathrm{Ru}(1)-\mathrm{Ct} 1$ & 1.823 \\
$\mathrm{Ru}(2)-\mathrm{Ct} 2$ & 1.881 & $\mathrm{Ru}(2)-\mathrm{Ct} 2$ & 1.795 \\
$\mathrm{Ru}(3)-\mathrm{Ct} 3$ & 1.711 & $\mathrm{Ru}(3)-\mathrm{Ct} 3$ & 3.042 \\
& & & \\
$\mathrm{Ru}(2)-\mathrm{Ru}(1)-\mathrm{Ru}(3)$ & 64.726 & $\mathrm{Ru}(2)-\mathrm{Ru}(1)-\mathrm{Ru}(3)$ & 63.586 \\
$\mathrm{Ru}(1)-\mathrm{Ru}(2)-\mathrm{Ru}(3)$ & 52.509 & $\mathrm{Ru}(1)-\mathrm{Ru}(2)-\mathrm{Ru}(3)$ & 57.963 \\
$\mathrm{Ru}(1)-\mathrm{Ru}(3)-\mathrm{Ru}(2)$ & 62.765 & $\mathrm{Ru}(1)-\mathrm{Ru}(3)-\mathrm{Ru}(2)$ & 58.451 \\
$\mathrm{Ru}(1)-\mathrm{Ru}(2)-\mathrm{P}(1)$ & 98.540 & $\mathrm{Ru}(1)-\mathrm{Ru}(3)-\mathrm{P}(1)$ & 122.832 \\
$\mathrm{Ru}(3)-\mathrm{Ru}(2)-\mathrm{P}(1)$ & 93.644 & $\mathrm{Ru}(2)-\mathrm{Ru}(3)-\mathrm{P}(1)$ & 113.700 \\
\hline $\mathrm{Relative} \mathrm{stabilities} \mathrm{at}$ & \multirow{2}{}{} & +25.96 \\
$298 \mathrm{~K}\left(\mathrm{kcal} \mathrm{mol}{ }^{-1}\right)$ & 0.00 & & + \\
\hline
\end{tabular}




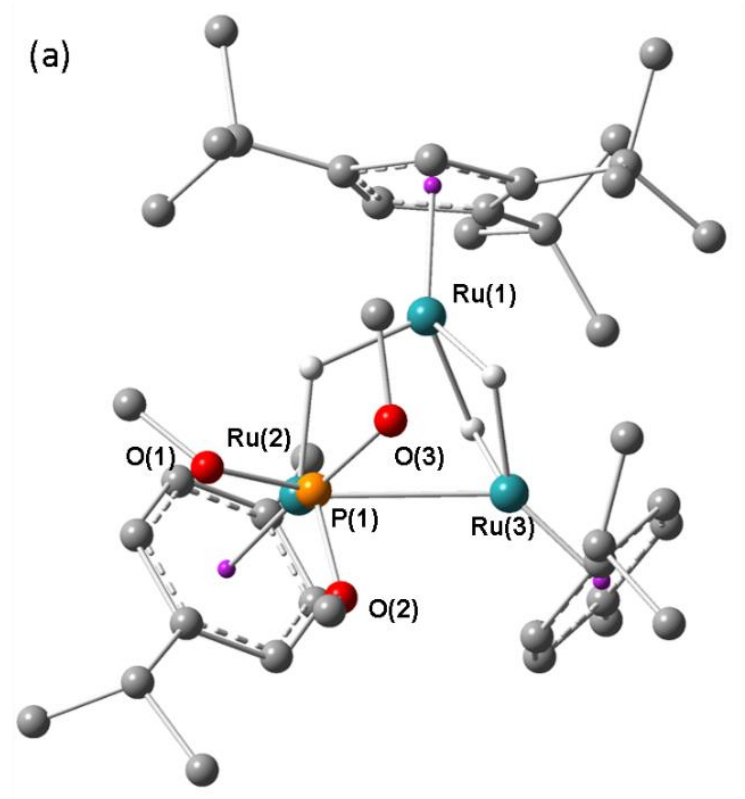

(b)

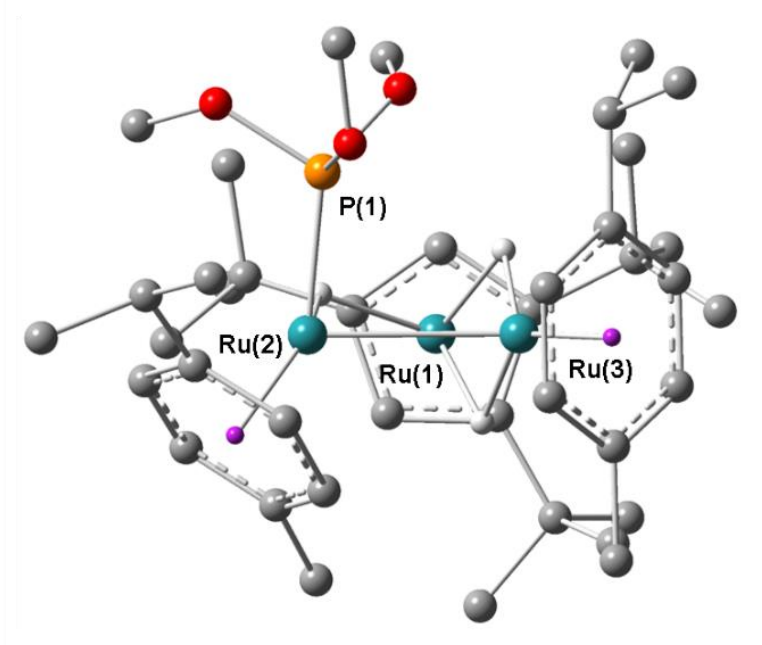

Figure S10. Optimized structures of $\left[(\mathrm{Cp} \Vdash \mathrm{Ru})\{(p \text {-cymene }) \mathrm{Ru}\}_{2}(\mu-\mathrm{H})_{3}\left\{\mathrm{P}(\mathrm{OMe})_{3}\right\}\right](\mathbf{8})$; (a) top view (b) side view $(\omega \mathrm{B} 97 \mathrm{XD} / \mathrm{SDD}(\mathrm{Ru}), 6-31++\mathrm{G}(\mathrm{C}, \mathrm{H}, \mathrm{O}$, and $\mathrm{P}))$. Hydrogen atoms except for attached to $\mathrm{Ru}$ atoms were omitted for clarity.

Table S8. Selected geometrical parameters for the optimized structures of $\mathbf{8}$ and observed data.

\begin{tabular}{|c||c||c||}
\hline & $\mathbf{8}$ (opt.) & $\mathbf{8}$ (obsd.) \\
\hline $\mathrm{Ru}(1)-\mathrm{Ru}(2)$ & 2.6448 & $2.6570(4)$ \\
$\mathrm{Ru}(1)-\mathrm{Ru}(3)$ & 2.9687 & $2.9804(4)$ \\
$\mathrm{Ru}(2)-\mathrm{Ru}(3)$ & 2.7822 & $2.7914(4)$ \\
$\mathrm{Ru}(3)-\mathrm{P}(1)$ & 2.1997 & $2.1971(9)$ \\
$\mathrm{P}(1)-\mathrm{O}(1)$ & 1.6666 & $1.637(2)$ \\
$\mathrm{P}(1)-\mathrm{O}(2)$ & 1.6341 & $1.615(3)$ \\
$\mathrm{P}(1)-\mathrm{O}(3)$ & 1.6364 & $1.617(3)$ \\
& & \\
$\mathrm{Ru}(2)-\mathrm{Ru}(1)-\mathrm{Ru}(3)$ & 59.088 & $59.029(9)$ \\
$\mathrm{Ru}(1)-\mathrm{Ru}(2)-\mathrm{Ru}(3)$ & 66.268 & $66.271(10)$ \\
$\mathrm{Ru}(1)-\mathrm{Ru}(3)-\mathrm{Ru}(2)$ & 54.645 & $54.700(9)$ \\
$\mathrm{Ru}(1)-\mathrm{Ru}(3)-\mathrm{P}(1)$ & 96.732 & $98.22(3)$ \\
$\mathrm{Ru}(2)-\mathrm{Ru}(3)-\mathrm{P}(1)$ & 84.692 & $85.47(2)$ \\
\hline
\end{tabular}

(a)
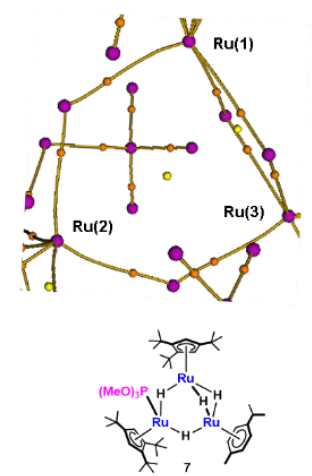

(b)
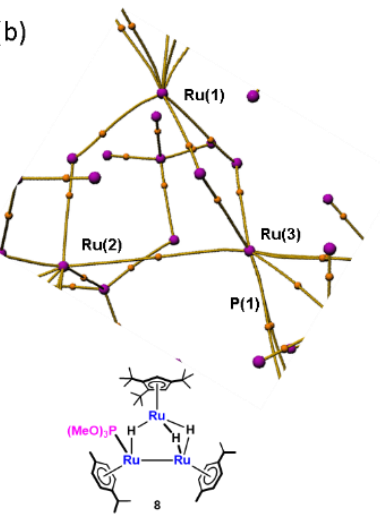

Figure S11. Enlarged view of the $\mathrm{Ru}_{3}$ core of (a) 7 and (b) 8, showing bond paths (orange lines), the bond critical points (small orange circles), and the ring critical points (small yellow circles). 

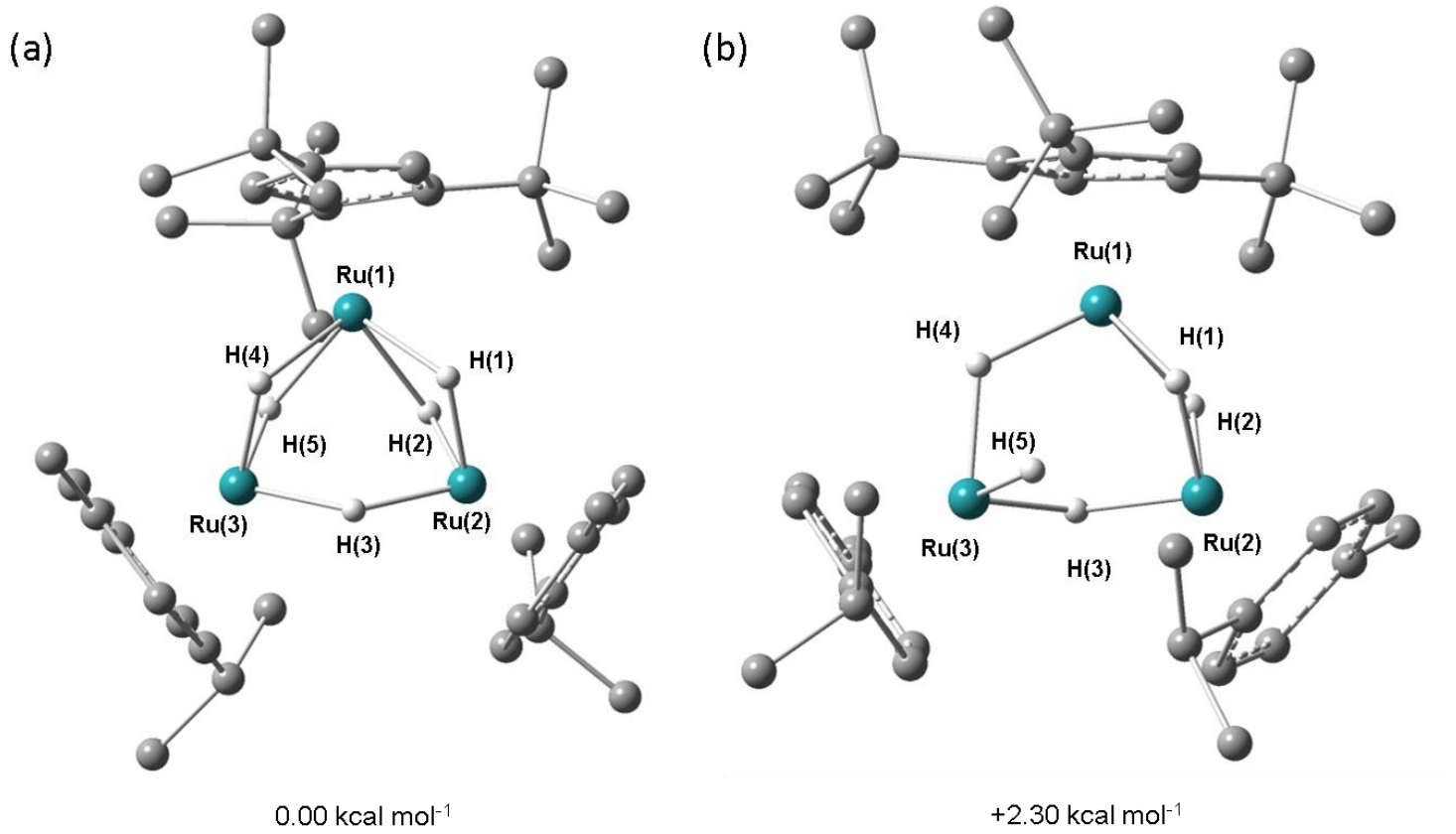

Figure S12. Optimized structures of $\left[(\mathrm{Cp}+\mathrm{Ru})\{(p \text {-cymene }) \mathrm{Ru}\}_{2}(\mu-\mathrm{H})_{5}\right]$ (a) 9a and (b) 9b $(\omega \mathrm{B} 97 \mathrm{XD} / \mathrm{SDD}(\mathrm{Ru}), 6-31++\mathrm{G}(\mathrm{C}$ and $\mathrm{H}))$. Hydrogen atoms except for attached to $\mathrm{Ru}$ atoms were omitted for clarity.

Table S9. Selected geometrical parameters for the optimized structures of $\mathbf{9 a}$ and $\mathbf{9 b}$, and those of observed data.

\begin{tabular}{c||c||c||c}
\hline & $9 a$ (opt.) & & 9b(opt.) \\
\hline $\mathrm{Ru}(1)-\mathrm{Ru}(2)$ & 2.7983 & $\mathrm{Ru}(1)-\mathrm{Ru}(2)$ & 2.7008 \\
$\mathrm{Ru}(1)-\mathrm{Ru}(3)$ & 2.7760 & $\mathrm{Ru}(1)-\mathrm{Ru}(3)$ & 3.0027 \\
$\mathrm{Ru}(2)-\mathrm{Ru}(3)$ & 3.0346 & $\mathrm{Ru}(2)-\mathrm{Ru}(3)$ & 2.9269 \\
$\mathrm{Ru}(1)-\mathrm{H}(1)$ & 1.8590 & $\mathrm{Ru}(1)-\mathrm{H}(1)$ & 1.8368 \\
$\mathrm{Ru}(1)-\mathrm{H}(2)$ & 2.0296 & $\mathrm{Ru}(1)-\mathrm{H}(2)$ & 1.8972 \\
$\mathrm{Ru}(1)-\mathrm{H}(4)$ & 1.8980 & $\mathrm{Ru}(1)-\mathrm{H}(4)$ & 1.8016 \\
$\mathrm{Ru}(1)-\mathrm{H}(5)$ & 1.9391 & $\mathrm{Ru}(1) \cdots \mathrm{H}(5)$ & 3.0172 \\
$\mathrm{Ru}(2)-\mathrm{H}(1)$ & 1.6510 & $\mathrm{Ru}(2)-\mathrm{H}(1)$ & 1.6935 \\
$\mathrm{Ru}(2)-\mathrm{H}(2)$ & 1.6379 & $\mathrm{Ru}(2)-\mathrm{H}(2)$ & 1.6712 \\
$\mathrm{Ru}(2)-\mathrm{H}(3)$ & 1.7116 & $\mathrm{Ru}(2)-\mathrm{H}(3)$ & 1.7331 \\
$\mathrm{Ru}(3)-\mathrm{H}(3)$ & 1.7275 & $\mathrm{Ru}(2) \cdots \mathrm{H}(5)$ & 2.7089 \\
$\mathrm{Ru}(3)-\mathrm{H}(4)$ & 1.6596 & $\mathrm{Ru}(3)-\mathrm{H}(3)$ & 1.7066 \\
$\mathrm{Ru}(3)-\mathrm{H}(5)$ & 1.6557 & $\mathrm{Ru}(3)-\mathrm{H}(4)$ & 1.6750 \\
& & $\mathrm{Ru}(3)-\mathrm{H}(5)$ & 1.57671 \\
$\mathrm{Ru}(2)-\mathrm{Ru}(1)-\mathrm{Ru}(3)$ & 65.967 & & 61.481 \\
$\mathrm{Ru}(1)-\mathrm{Ru}(2)-\mathrm{Ru}(3)$ & 56.663 & $\mathrm{Ru}(2)-\mathrm{Ru}(1)-\mathrm{Ru}(3)$ & 64.345 \\
$\mathrm{Ru}(1)-\mathrm{Ru}(3)-\mathrm{Ru}(2)$ & 57.370 & $\mathrm{Ru}(1)-\mathrm{Ru}(2)-\mathrm{Ru}(3)$ & 54.174 \\
\hline $\mathrm{Relative} \mathrm{stabilities} \mathrm{at}$ & \multirow{2}{*}{0.00} & & +2.30 \\
298 K (kcal mol $\left.{ }^{-1}\right)$ & & & \\
\hline
\end{tabular}




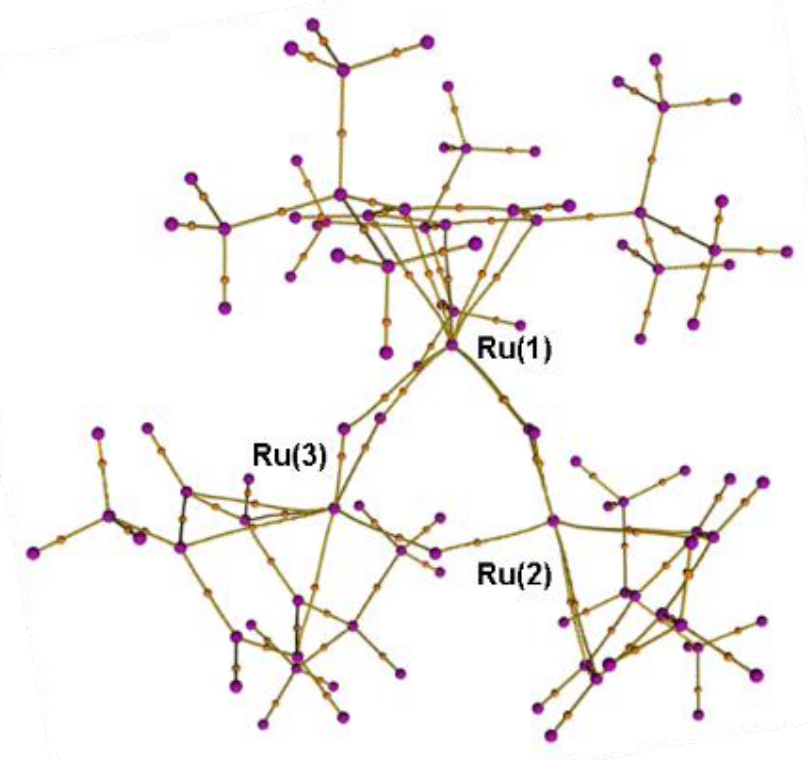

Figure S13. Optimized structures of 9a, showing bond paths (orange lines), the bond critical points (small orange circles), and the ring critical points (small yellow circles). 


\section{NMR spectra of compounds}

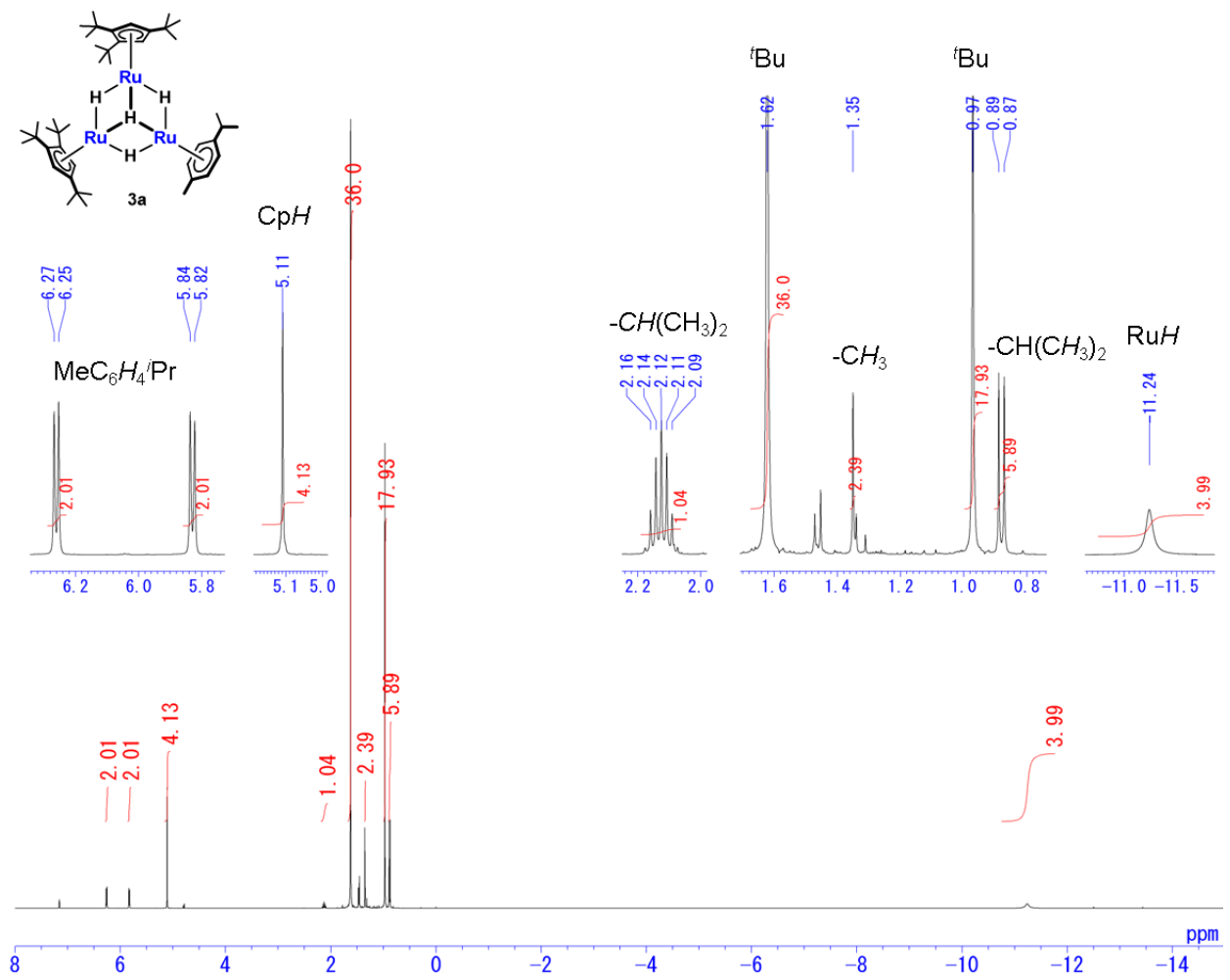

Figure S14. ${ }^{1} \mathrm{H}$ NMR spectrum of $\mathbf{3 a}\left(400 \mathrm{MHz}, \mathrm{C}_{6} \mathrm{D}_{6}, 25^{\circ} \mathrm{C}\right)$.

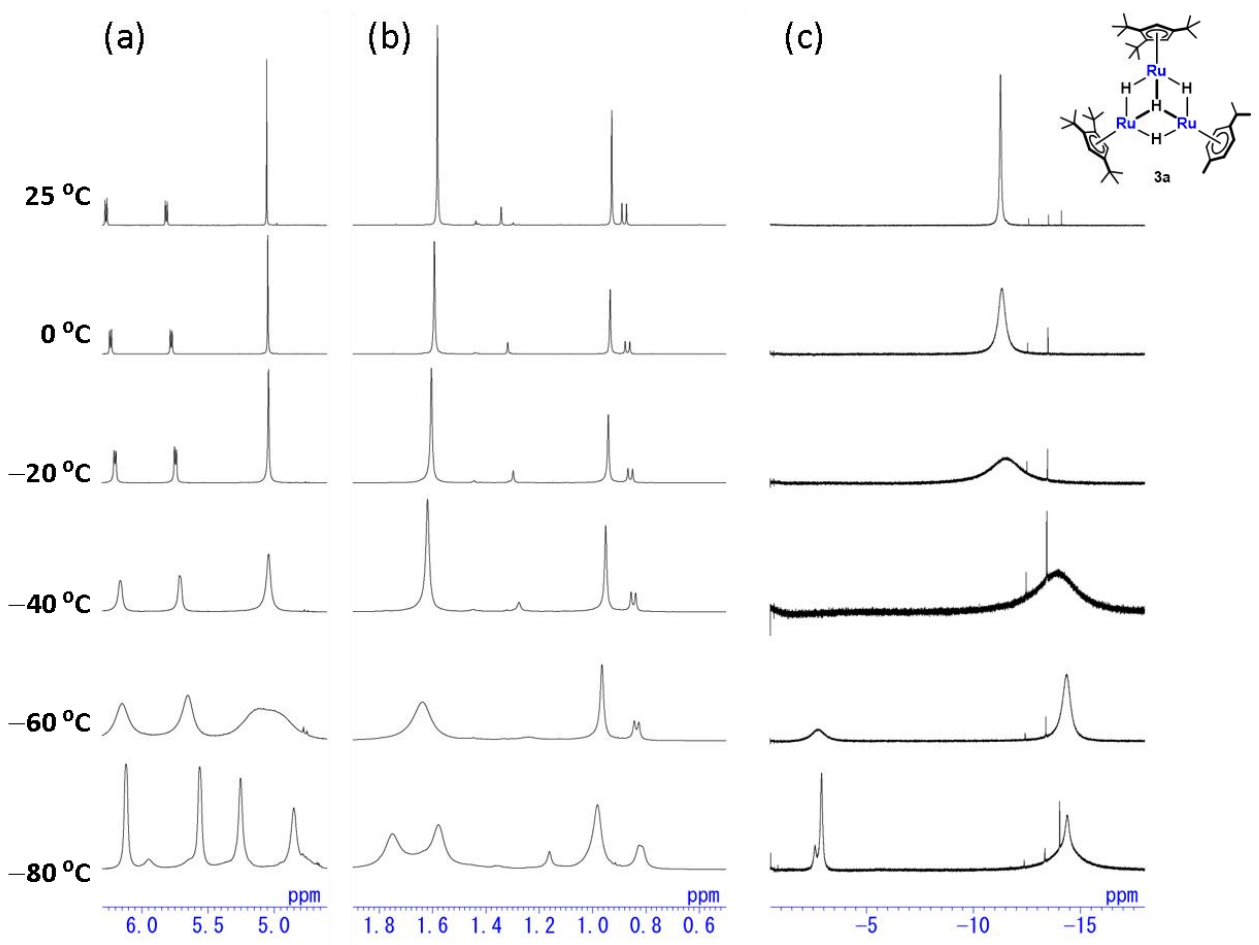

Figure S15. Variable temperature ${ }^{1} \mathrm{H}$ NMR spectra of 3a showing (a) $\mathrm{CpH}$ and arene- $\mathrm{H}$, (b) ${ }^{t} \mathrm{Bu}$, and (c) hydrido regions $\left(400 \mathrm{MHz}\right.$, toluene- $\left.d_{8}\right)$. 


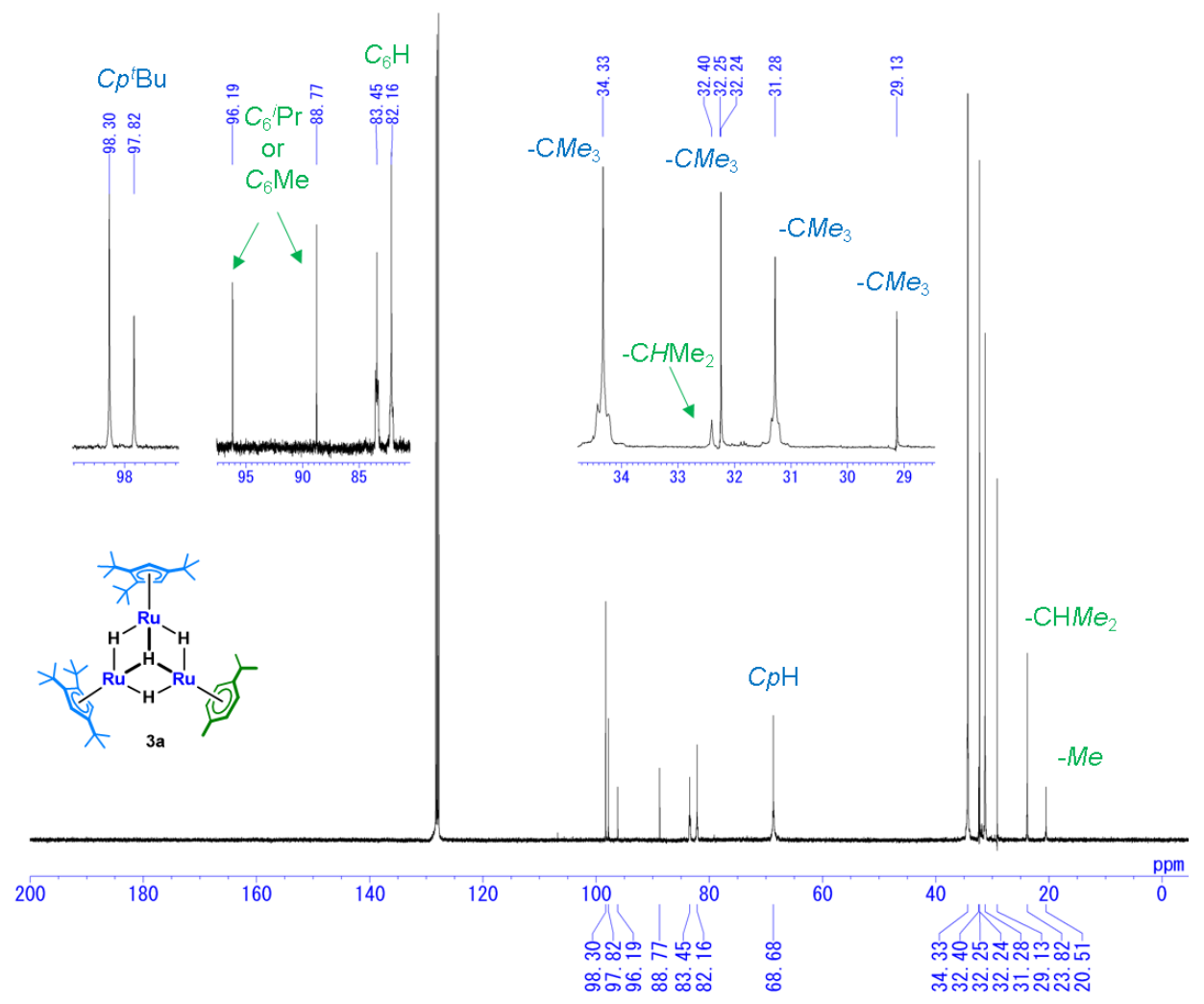

Figure S16. ${ }^{13} \mathrm{C}\left\{{ }^{1} \mathrm{H}\right\}$ NMR spectrum of $\mathbf{3 a}\left(100 \mathrm{MHz}, \mathrm{C}_{6} \mathrm{D}_{6}, 25{ }^{\circ} \mathrm{C}\right)$.

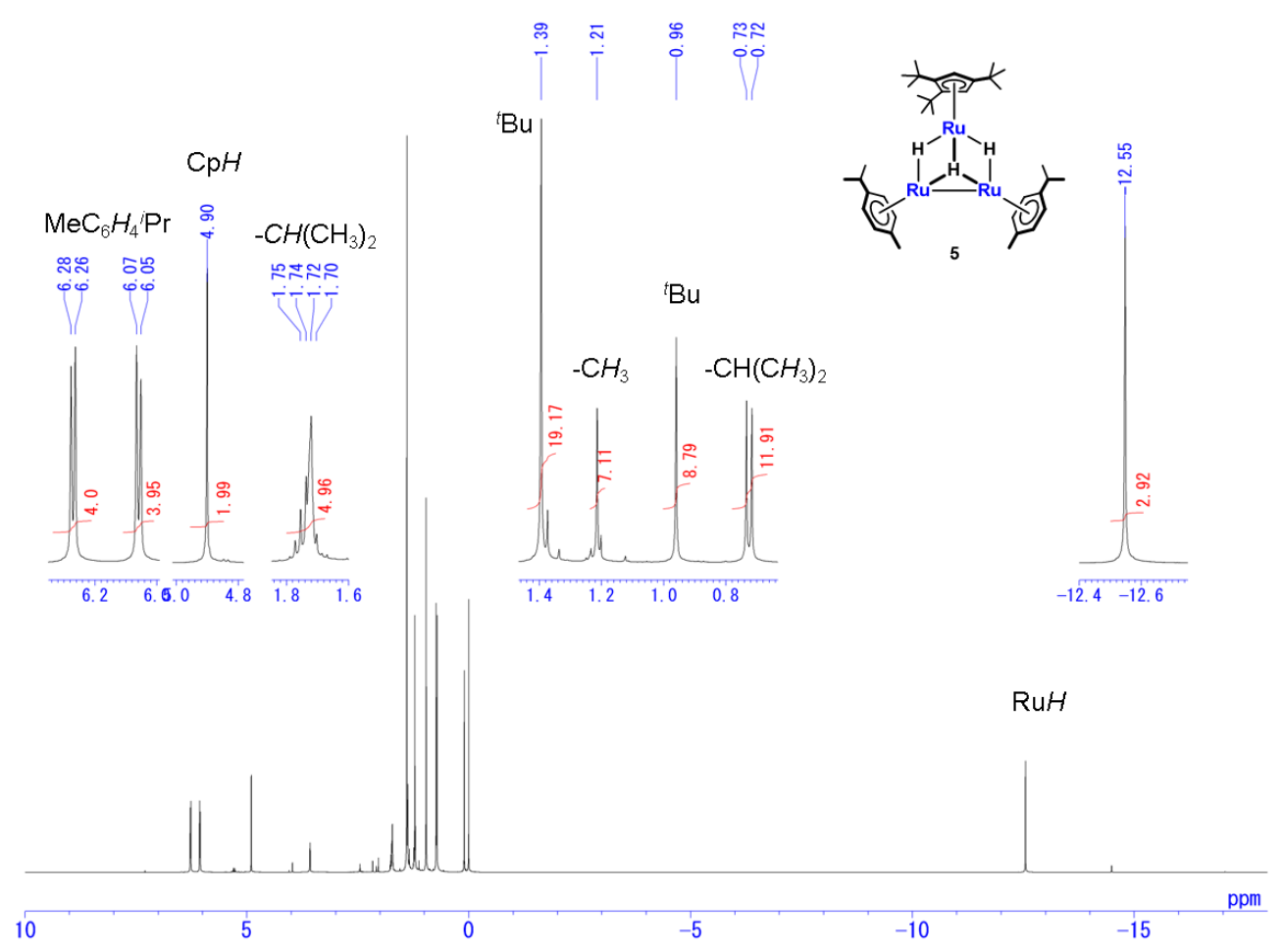

Figure S17. ${ }^{1} \mathrm{H}$ NMR spectrum of $5\left(400 \mathrm{MHz}, \mathrm{THF}-d_{8}, 25^{\circ} \mathrm{C}\right)$. 
(a)

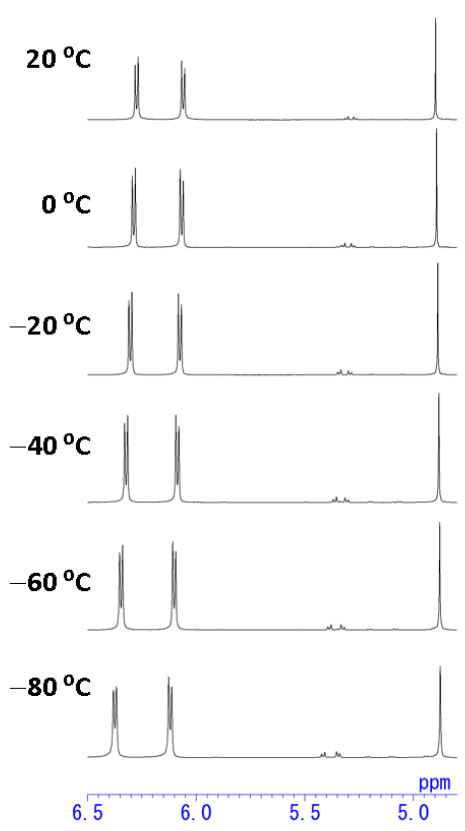

(b)

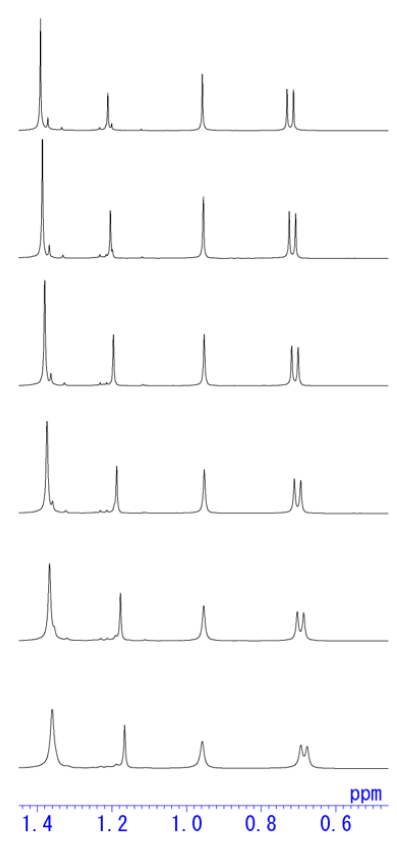

(c)
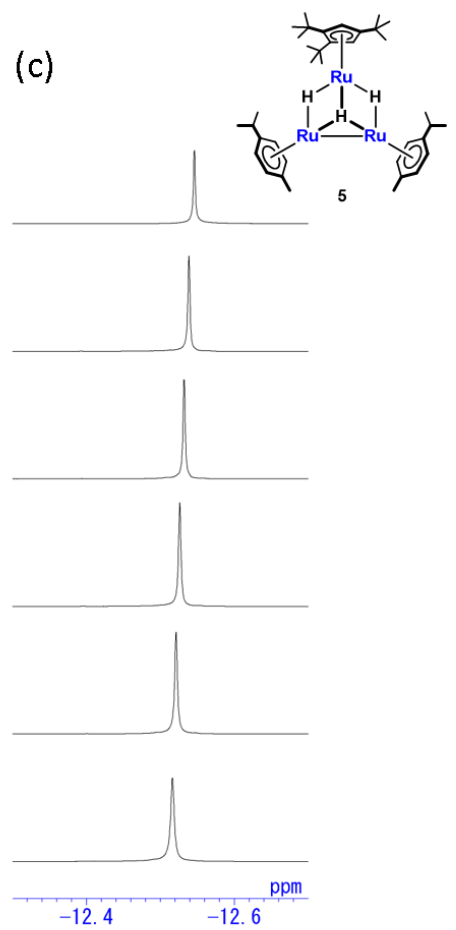

Figure S18. Variable temperature ${ }^{1} \mathrm{H}$ NMR spectra of 5 showing (a) $\mathrm{CpH}$ and arene- $\mathrm{H}$, (b) ${ }^{t} \mathrm{Bu}$, and (c) hydrido regions ( $\left.400 \mathrm{MHz}, \mathrm{THF}-d_{8}\right)$.

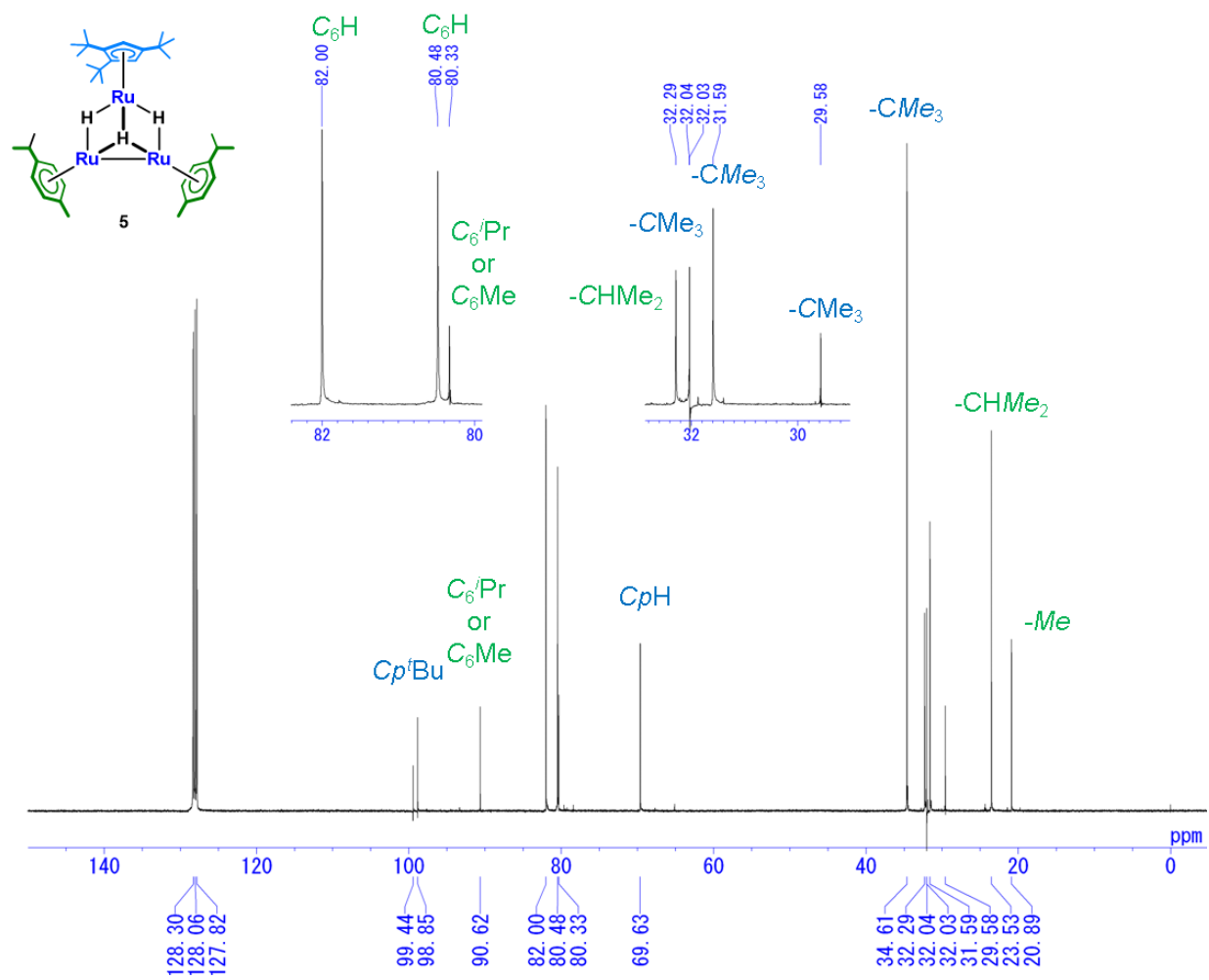

Figure S19. ${ }^{13} \mathrm{C}\left\{{ }^{1} \mathrm{H}\right\}$ NMR spectrum of $5\left(100 \mathrm{MHz}, \mathrm{C}_{6} \mathrm{D}_{6}, 25{ }^{\circ} \mathrm{C}\right)$. 


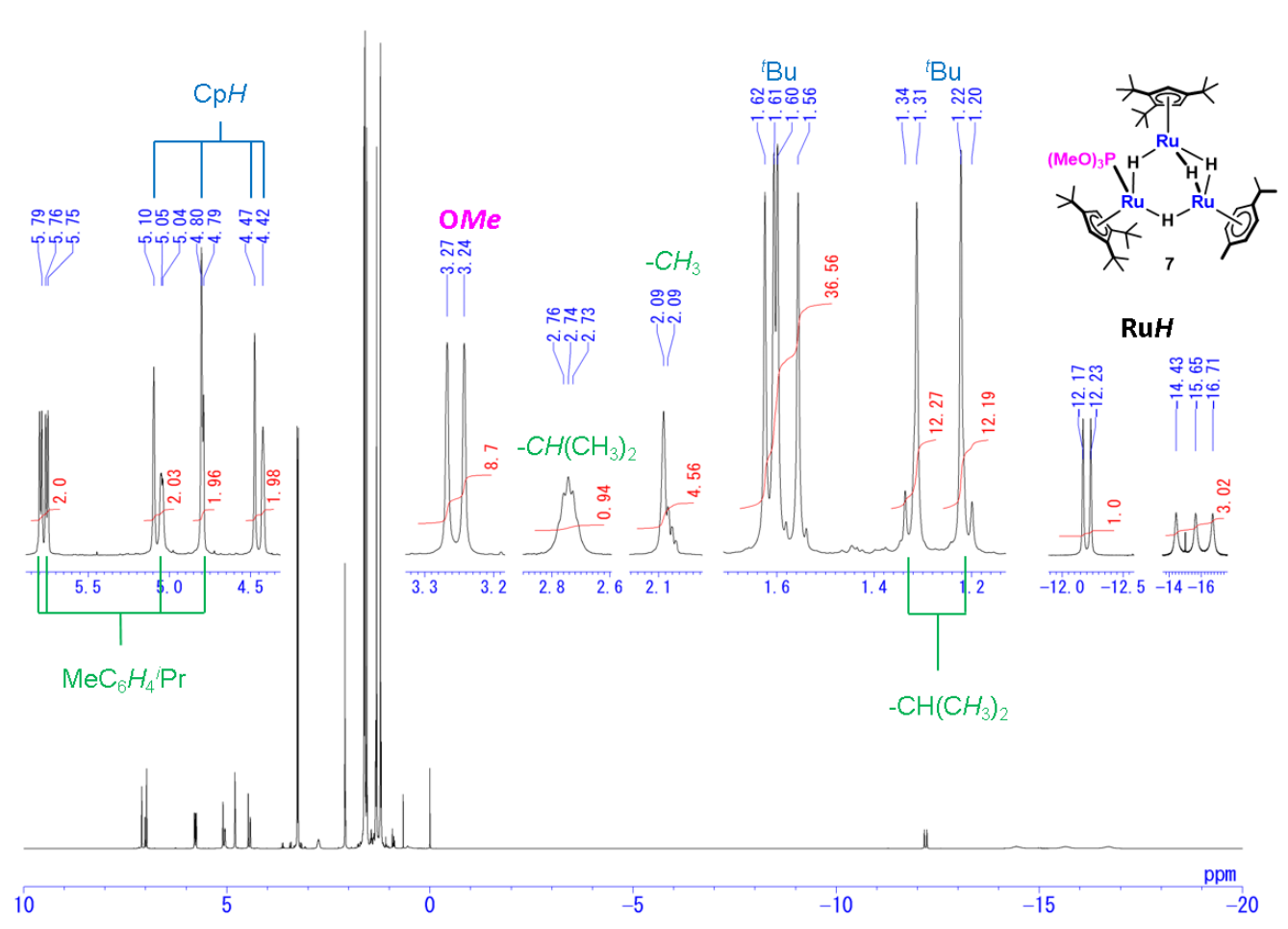

Figure S20. ${ }^{1} \mathrm{H}$ NMR spectrum of $7\left(400 \mathrm{MHz}\right.$, toluene- $\left.d_{8}, 20^{\circ} \mathrm{C}\right)$.
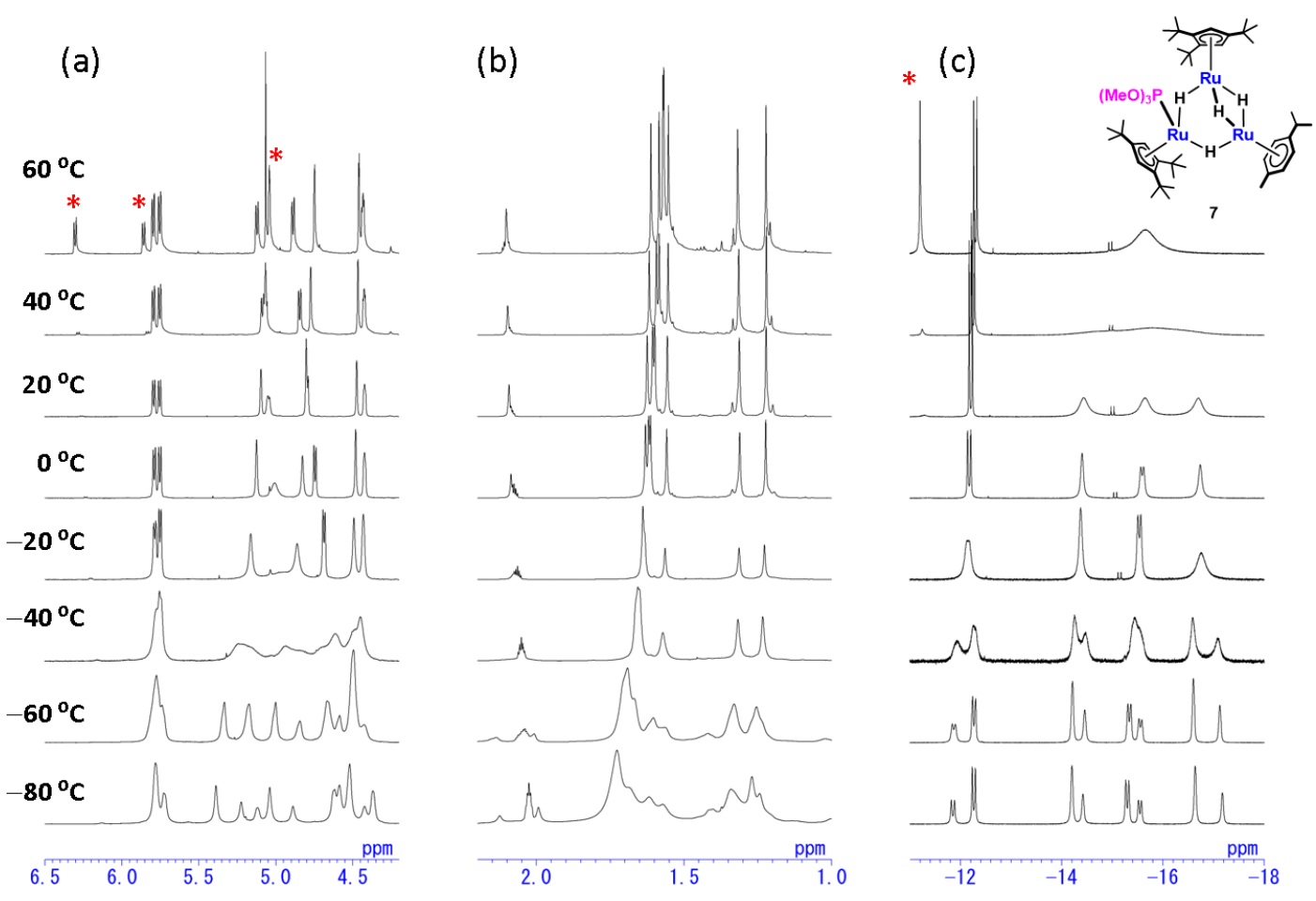

Figure S21. Variable temperature ${ }^{1} \mathrm{H}$ NMR spectra of 7 showing (a) $\mathrm{CpH}$ and arene- $\mathrm{H}$, (b) ${ }^{t} \mathrm{Bu}$, and (c) hydrido regions $\left(400 \mathrm{MHz}\right.$, toluene- $\left.d_{8}\right)$. Signals with an asterisk are derived from regenerated 3a upon heating at $60^{\circ} \mathrm{C}$. 


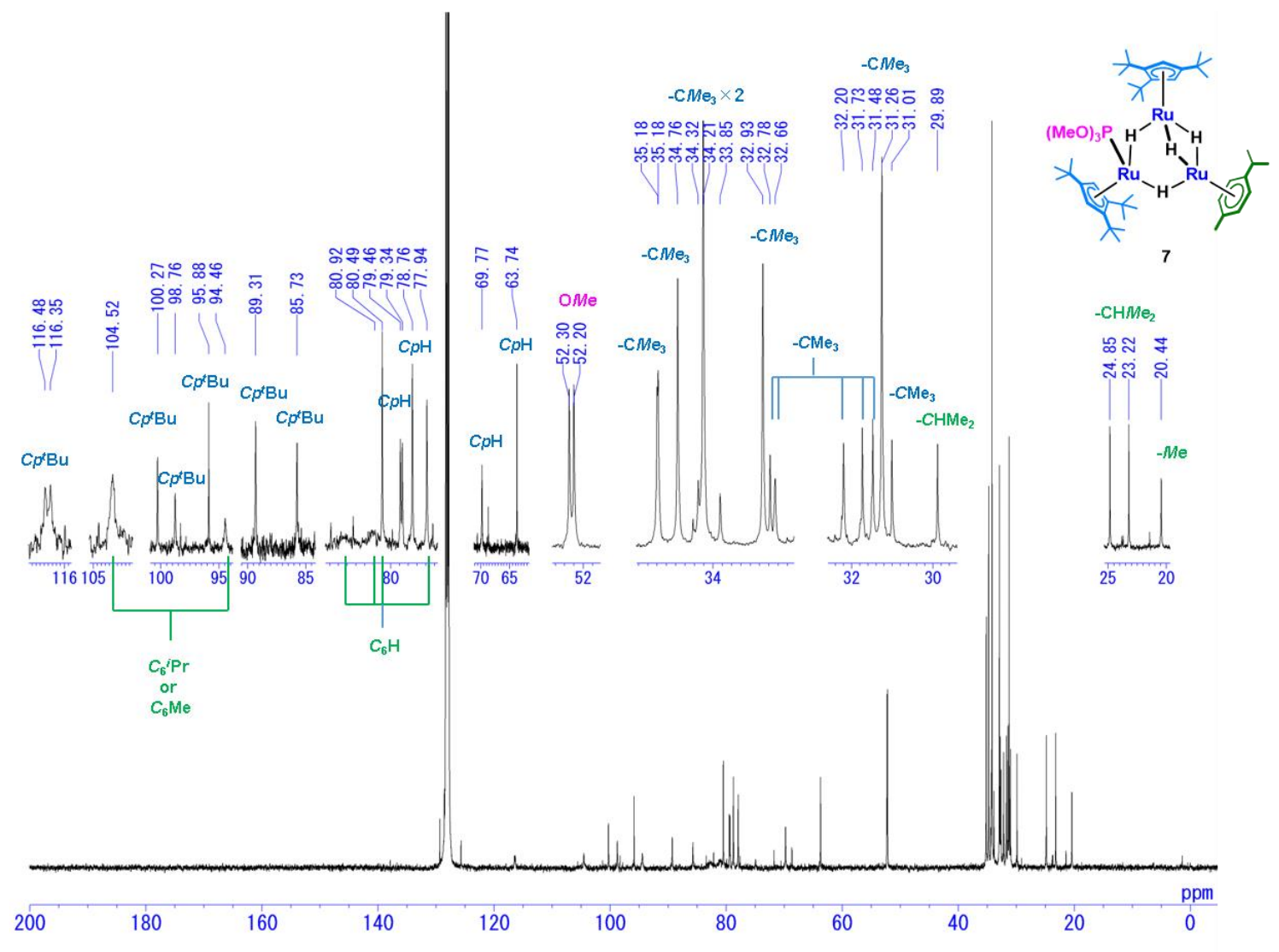

Figure S22. ${ }^{13} \mathrm{C}\left\{{ }^{1} \mathrm{H}\right\}$ NMR spectrum of $7\left(100 \mathrm{MHz}, \mathrm{C}_{6} \mathrm{D}_{6}, 25^{\circ} \mathrm{C}\right)$.

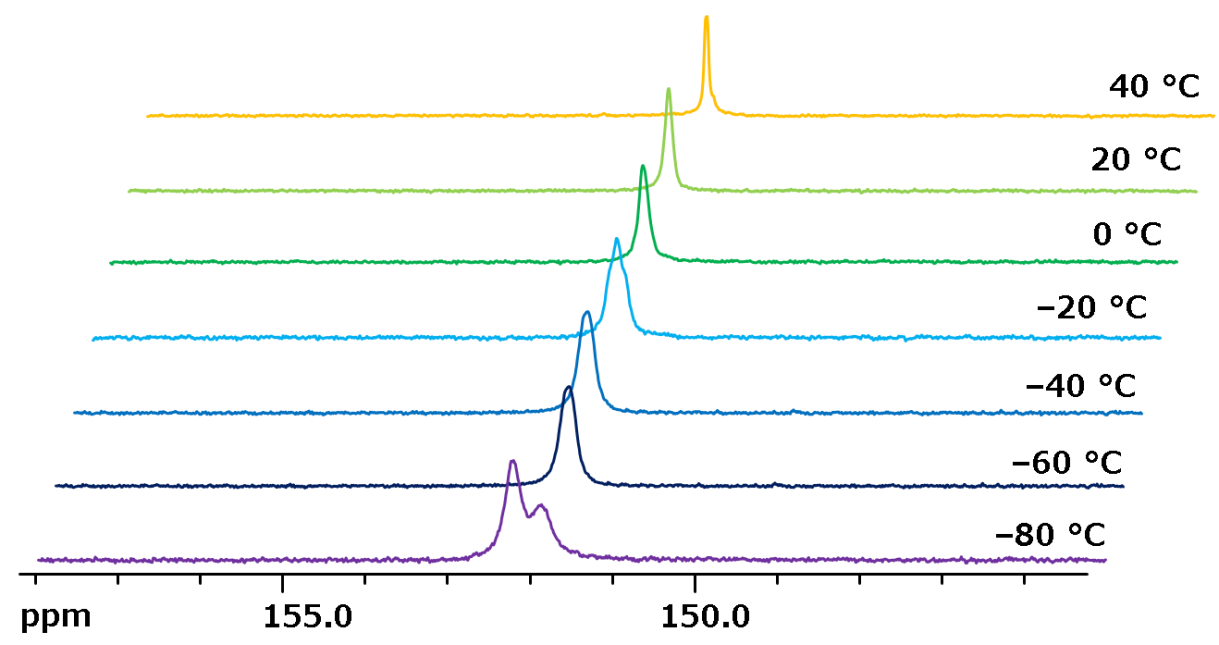

Figure S23. Variable temperature ${ }^{31} \mathrm{P}\left\{{ }^{1} \mathrm{H}\right\}$ NMR spectra of $7\left(161 \mathrm{MHz}, \mathrm{C}_{6} \mathrm{D}_{6}\right)$. 


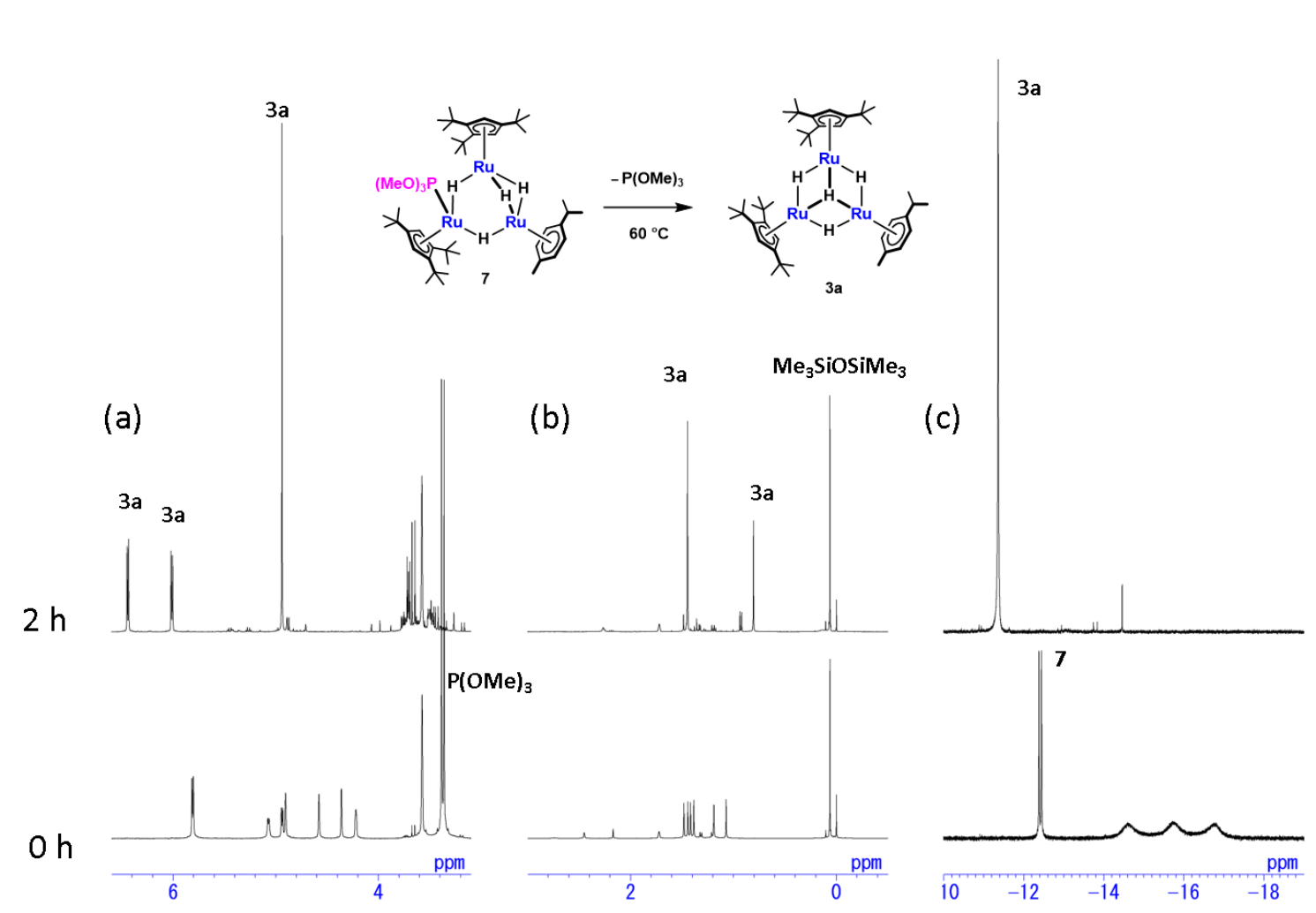

Figure S24. ${ }^{1} \mathrm{H}$ NMR spectrum of the products obtained from the thermolysis of 7 at $60{ }^{\circ} \mathrm{C}$ for $2 \mathrm{~h}$ (400 MHz, THF- $d_{8}, 25^{\circ} \mathrm{C}$ ).

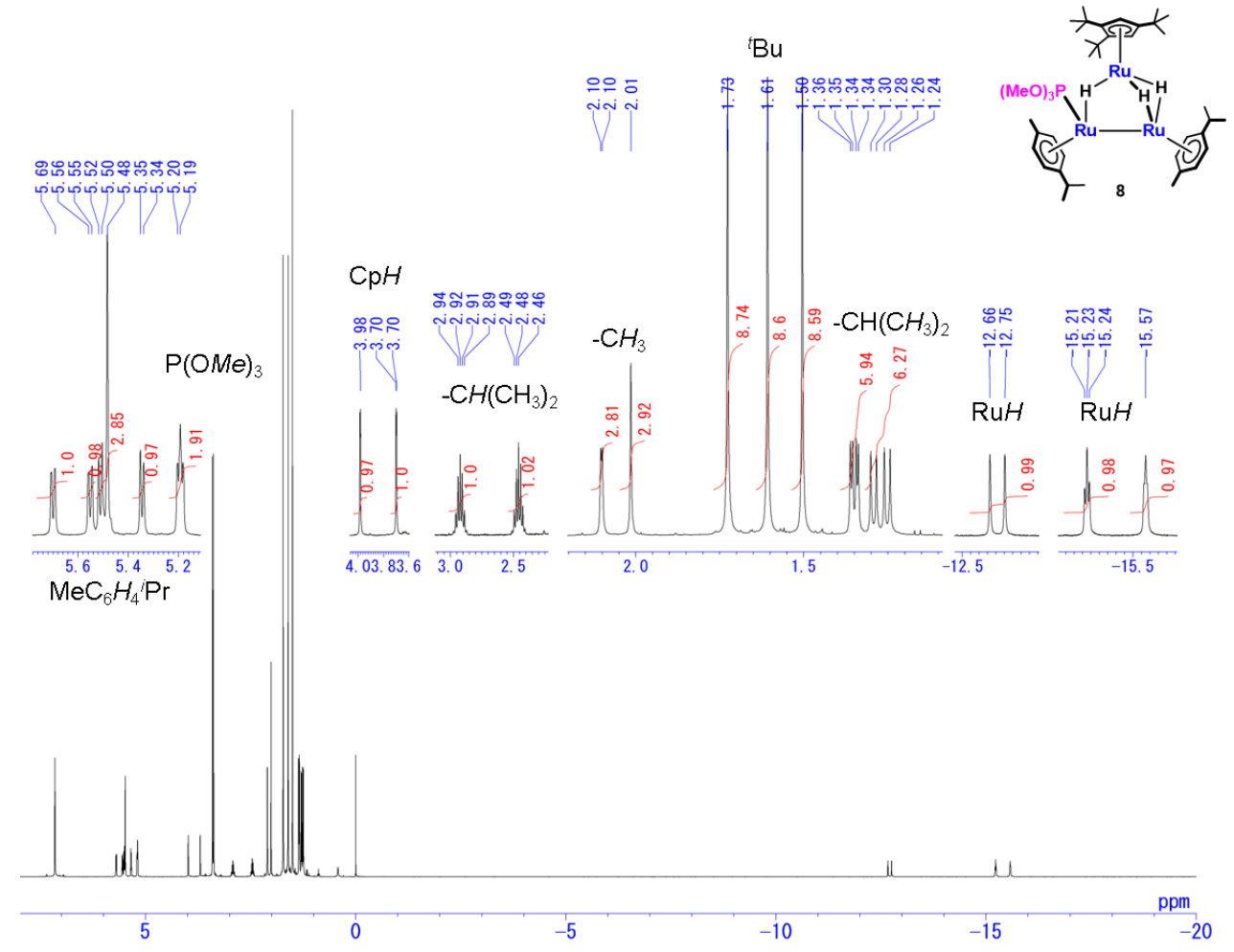

Figure S25. ${ }^{1} \mathrm{H}$ NMR spectrum of $8\left(400 \mathrm{MHz}, \mathrm{C}_{6} \mathrm{D}_{6}, 25^{\circ} \mathrm{C}\right)$. 

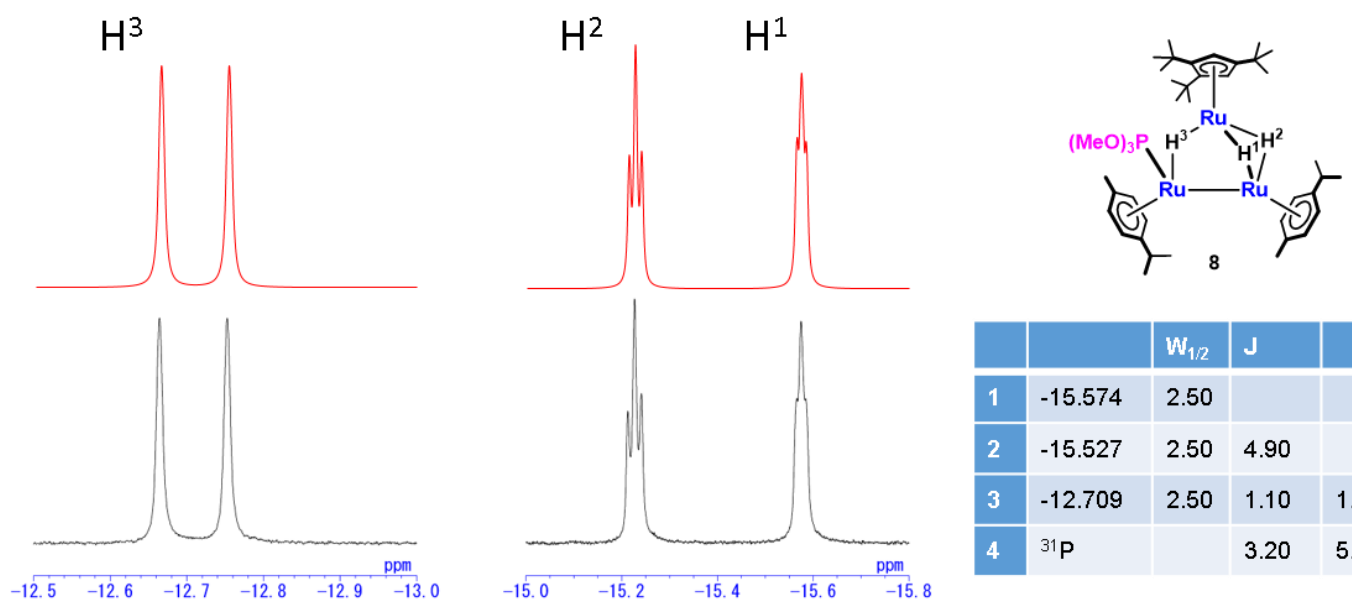

\begin{tabular}{|l|l|l|l|l|l|}
\hline & & W $_{12}$ & $\mathrm{~J}$ & & \\
\hline 1 & -15.574 & 2.50 & & & \\
\hline 2 & -15.527 & 2.50 & 4.90 & & \\
\hline 3 & -12.709 & 2.50 & 1.10 & 1.00 & \\
\hline 4 & $31 \mathrm{P}$ & & 3.20 & 5.60 & 35.0 \\
\hline
\end{tabular}

Figure S26. ${ }^{1} \mathrm{H}$ NMR Shapes of the hydrido signals of $\mathbf{8}$ (below) and results of spectral simulations (above).

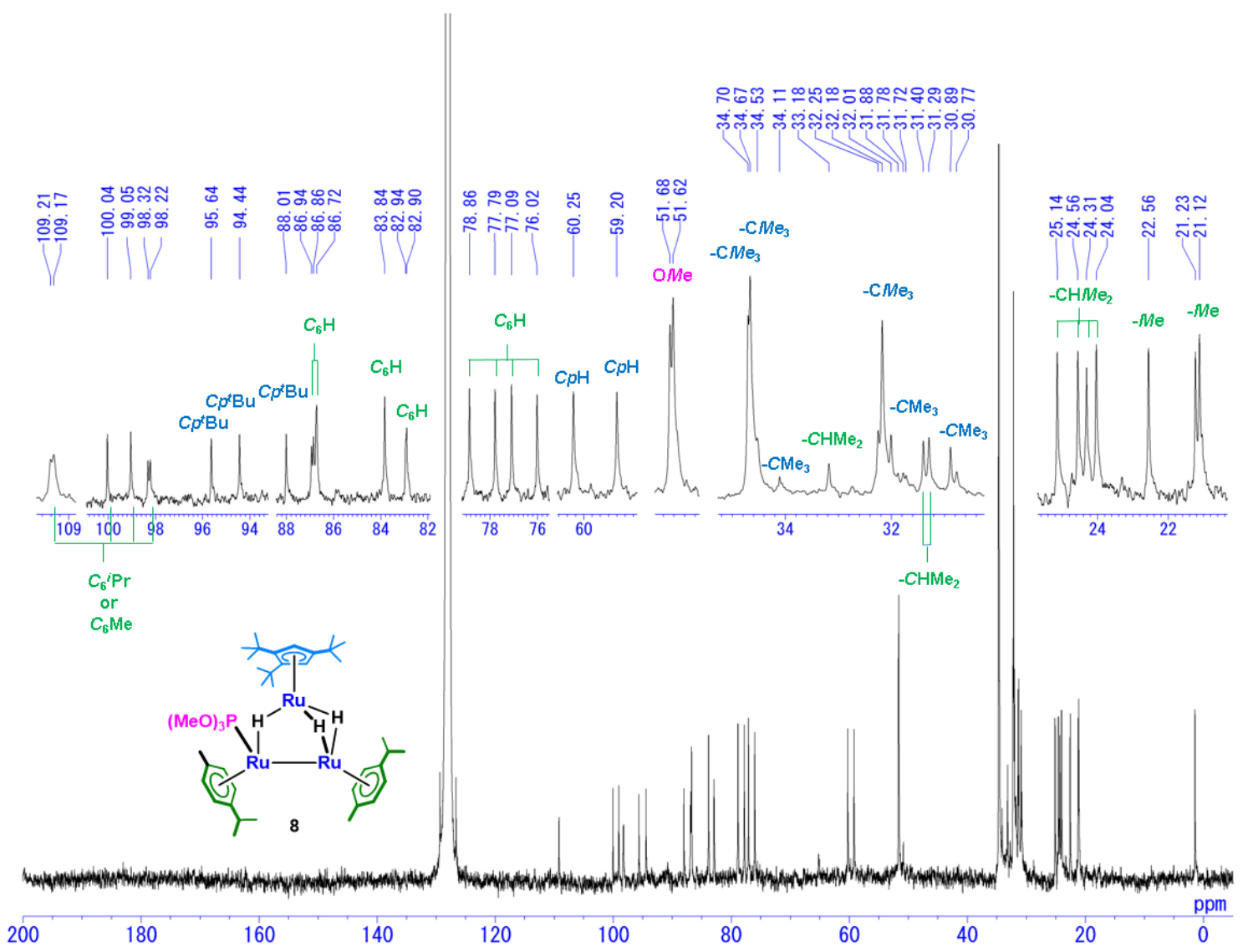

Figure S27. ${ }^{13} \mathrm{C}\left\{{ }^{1} \mathrm{H}\right\}$ NMR spectrum of $\mathbf{8}\left(100 \mathrm{MHz}, \mathrm{C}_{6} \mathrm{D}_{6}, 25{ }^{\circ} \mathrm{C}\right)$. 


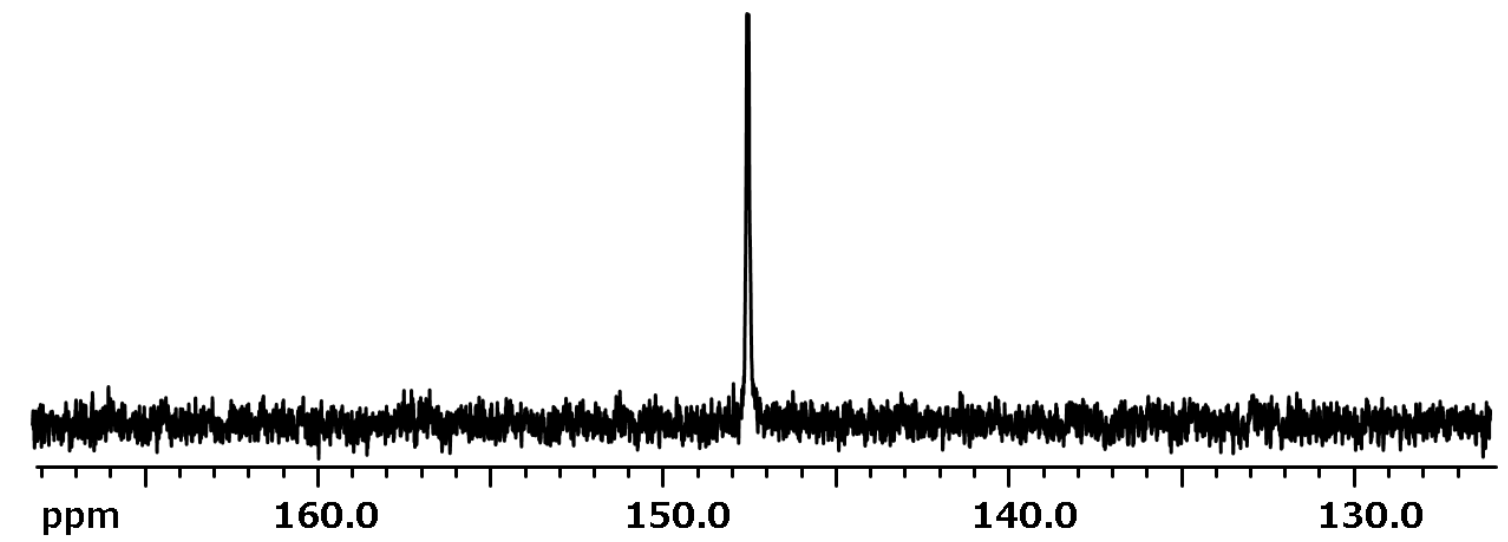

Figure S28. ${ }^{31} \mathrm{P}\left\{{ }^{1} \mathrm{H}\right\} \mathrm{NMR}$ spectra of $8\left(161 \mathrm{MHz}, \mathrm{C}_{6} \mathrm{D}_{6}, 25{ }^{\circ} \mathrm{C}\right)$.

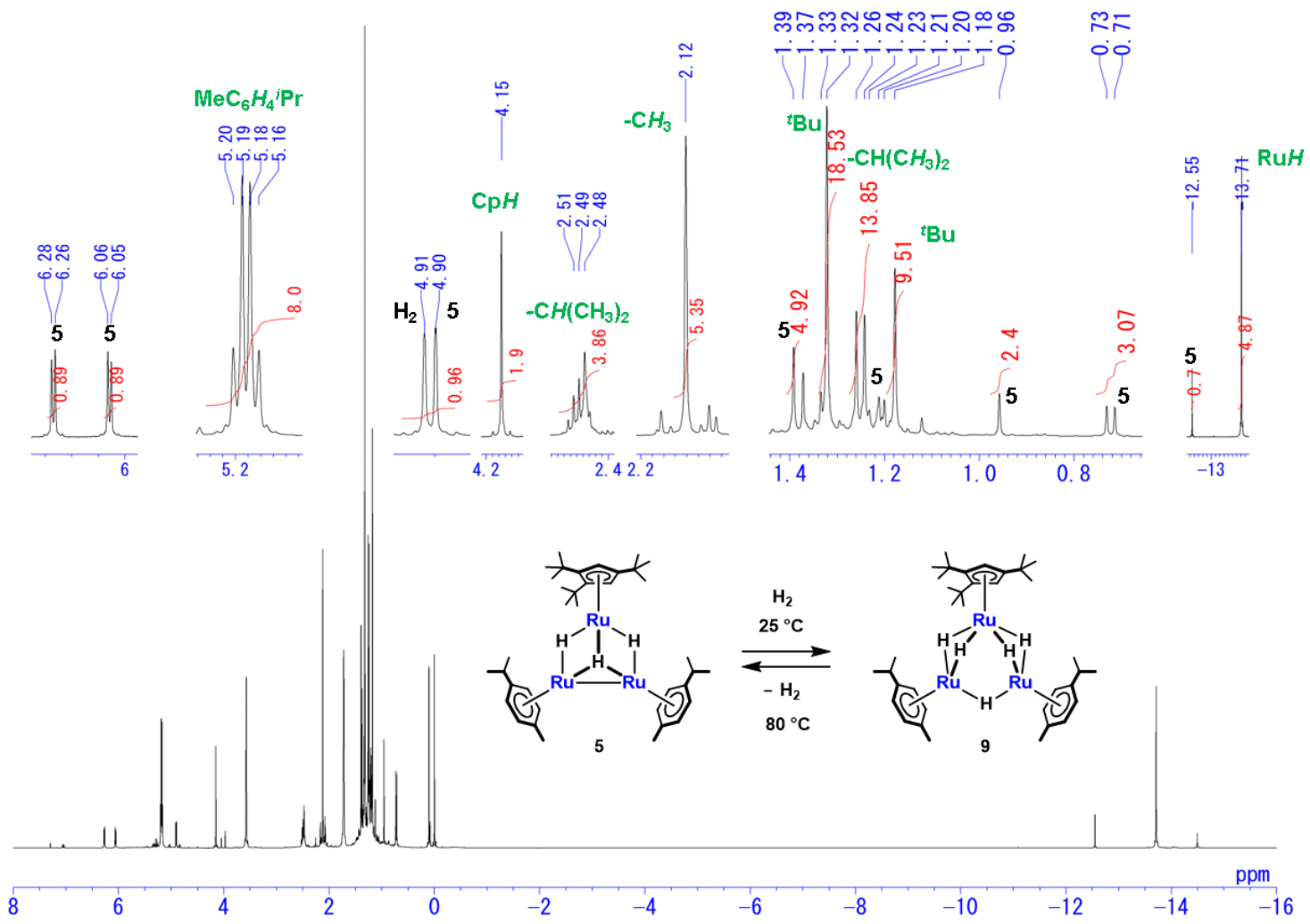

Figure S29. ${ }^{1} \mathrm{H}$ NMR spectrum of the equilibrated mixture of 5 and 9 (400 MHz, THF- $\left.d_{8}, 25{ }^{\circ} \mathrm{C}\right)$. 

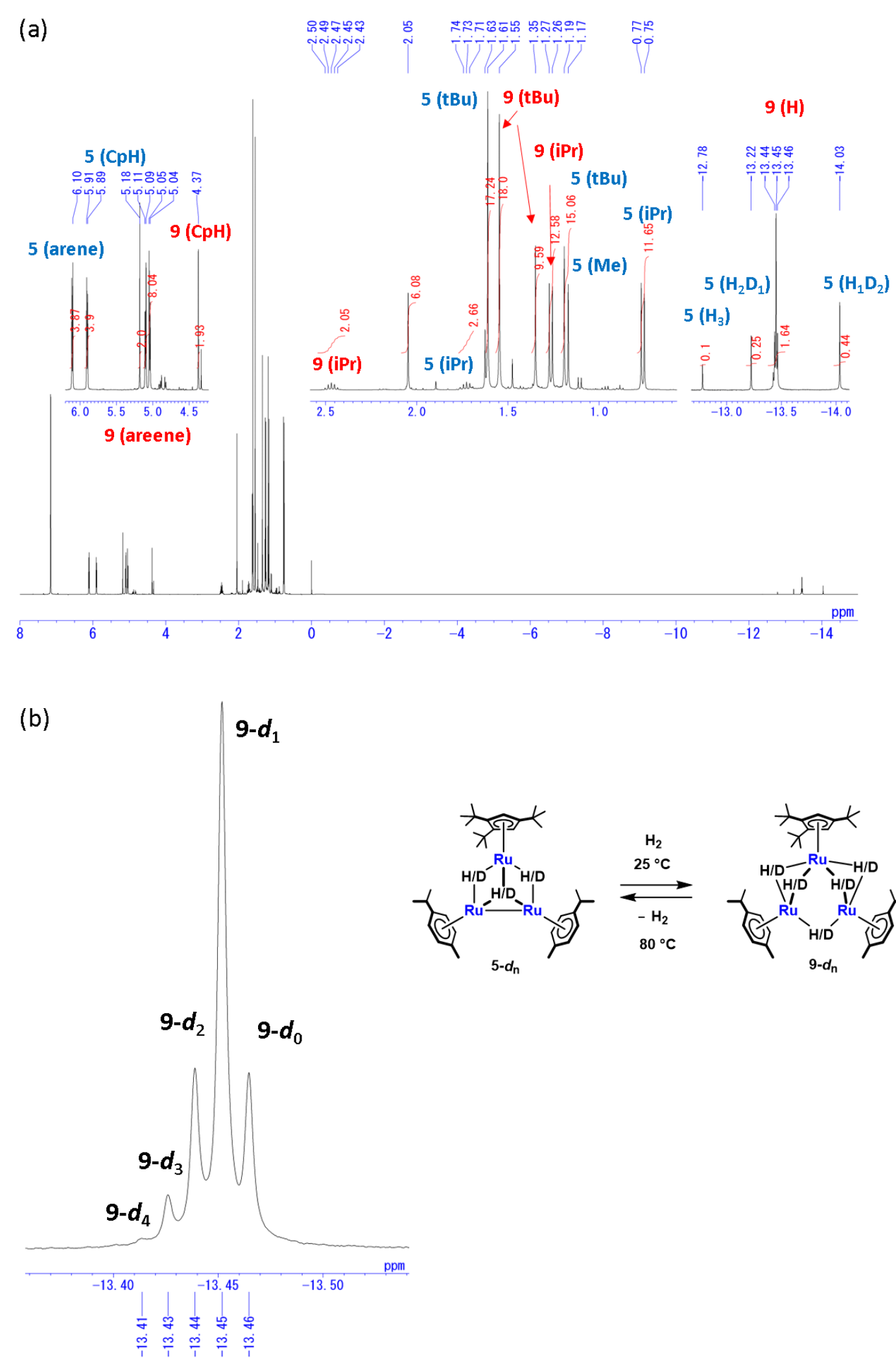

Figure S30. (a) ${ }^{1} \mathrm{H}$ NMR spectrum of the equilibrated mixture of 5 and 9 recorded in $\mathrm{C}_{6} \mathrm{D}_{6}$ and (b) hydrido signals of the isotopomers of $9\left(400 \mathrm{MHz}, \mathrm{C}_{6} \mathrm{D}_{6}, 25^{\circ} \mathrm{C}\right)$. 


\section{Cyclic voltammograms of $3 a, 5$, and 6}
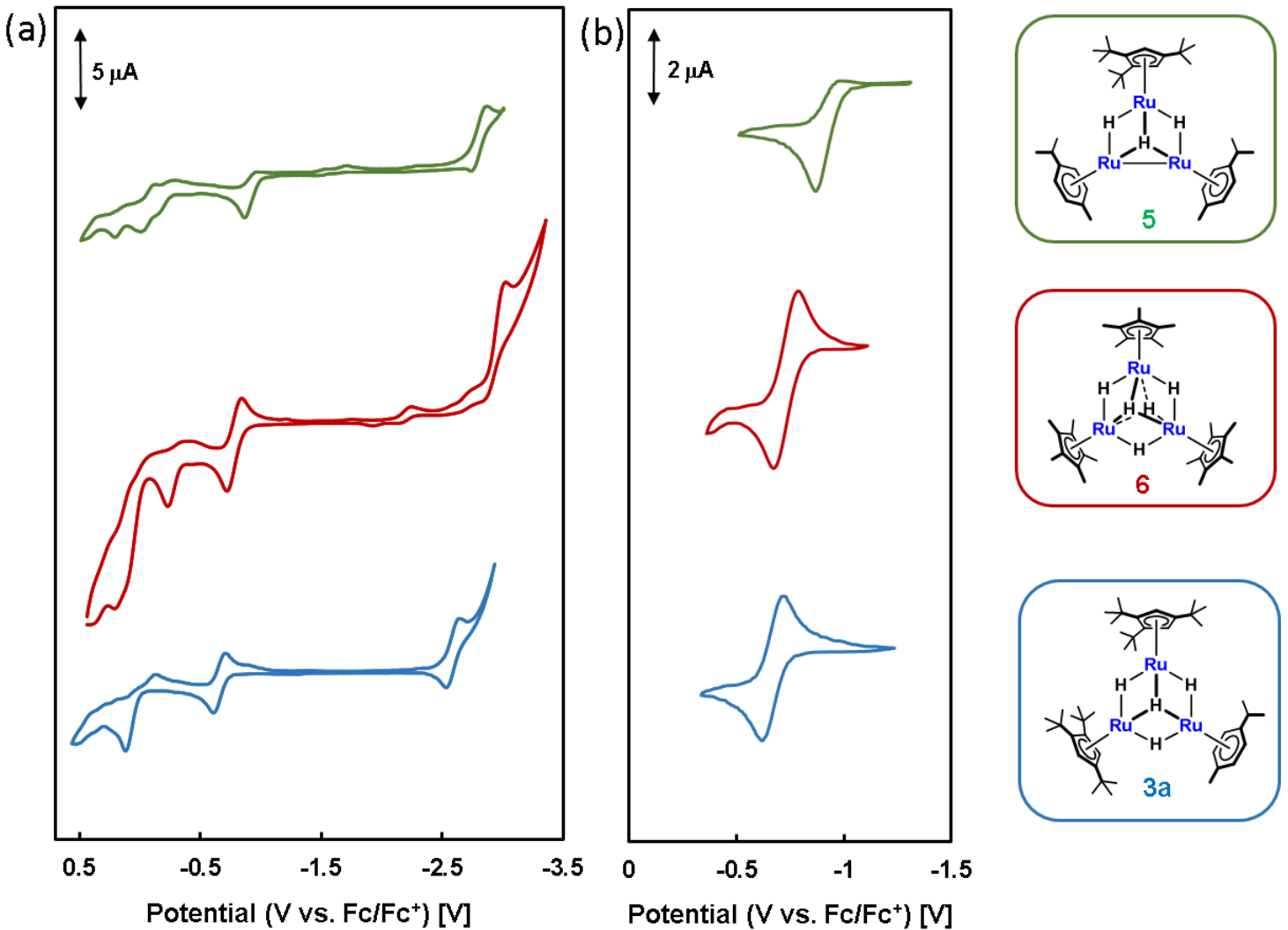

Figure S31. Cyclic voltammograms of 3a, 5, and $\mathbf{6}$ measured in THF at $25^{\circ} \mathrm{C}$; (a) range between -3.3 and $+0.5 \mathrm{~V}$, (b) range between -1.2 and $-0.3 \mathrm{~V}$ showing the redox at $0 /+1$. Conditions: electrolyte, $0.1 \mathrm{M}\left[\mathrm{NBu}_{4}\right]\left(\mathrm{PF}_{6}\right)$; working electrode, $\mathrm{Pt}$; counter electrode, $\mathrm{Pt}$, scan rate $50 \mathrm{mV} / \mathrm{s}$. Potentials are referenced to $\mathrm{Cp}_{2} \mathrm{Fe} /\left[\mathrm{Cp}_{2} \mathrm{Fe}\right]^{+}$. 